\title{
Bioresponsive Functional Phenylboronic Acid-Based Delivery System as an Emerging Platform for Diabetic Therapy
}

This article was published in the following Dove Press journal: International Journal of Nanomedicine

\author{
Qiong $M a^{\prime}$ \\ Xi Zhao' \\ Anhua Shi ${ }^{1}$ \\ Junzi $W u^{1,2}$ \\ 'The Key Laboratory of Microcosmic \\ Syndrome Differentiation, Education \\ Department of Yunnan, Yunnan \\ University of Chinese Medicine, Kunming, \\ Yunnan 650500, People's Republic of \\ China; ${ }^{2}$ Department of Medical Biology, \\ College of Basic Medicine, Yunnan \\ University of Chinese Medicine, Kunming, \\ Yunnan 650500, People's Republic of \\ China
}

\begin{abstract}
The glucose-sensitive self-adjusting drug delivery system simulates the physiological model of the human pancreas-secreting insulin and then precisely regulates the release of hypoglycemic drugs and controls the blood sugar. Thus, it has good application prospects in the treatment of diabetes. Presently, there are three glucose-sensitive drug systems: phenylboronic acid (PBA) and its derivatives, concanavalin A (Con A), and glucose oxidase (GOD). Among these, the glucose-sensitive polymer carrier based on PBA has the advantages of better stability, long-term storage, and reversible glucose response, and the loading of insulin in it can achieve the controlled release of drugs in the human environment. Therefore, it has become a research hotspot in recent years and has been developed very rapidly. In order to further carry out a follow-up study, we focused on the development process, performance, and application of PBA and its derivatives-based glucose-sensitive polymer drug carriers, and the prospects for the development of this field.
\end{abstract}

Keywords: phenylboronic acid, glucose sensitive, diabetes

\section{Introduction}

Diabetes mellitus is a group of clinical metabolic syndrome characterized by the disorder of glycometabolism, caused by genetic and environmental factors. It is an important branch of endocrine disease. ${ }^{1}$ With the rapid increase in the number of young DM patients, the treatment of diabetes is imminent. Diabetes mainly pertains to insulin secretion abnormality and/or insulin resistance, and hence, the human body cannot regulate the blood glucose concentration, cause carbohydrate, water, fat, and protein, and metabolism barrier. Over the years, it has a great impact on the body's blood vessels, nerves, and skin, and causes several chronic complications, threatening human health.

At present, the main drug treatment for diabetes is insulin, which can effectively control the concentration of blood sugar in patients with diabetes. ${ }^{2}$ Supplementing exogenous insulin is the most direct and effective method to treat diabetes. ${ }^{3}$ However, due to the short half-life of insulin in the body, frequent injections of insulin are required; multiple injections a day may cause local tissue infections or paralysis and other side effects, such as nerve damage. This causes great physical and mental pain to the patient, and using this method for a prolonged period has poor tolerance and compliance to the treatment. ${ }^{4}$ Therefore, researchers are pursuing for a better way to mimic the body's pancreas for feedback and balance, an intelligent system that
Correspondence: Junzi Wu; Anhua Shi Yunnan University of Chinese Medicine No. 1076, Yuhua Road, Chenggong

District, Kunming, Yunnan 650500,

People's Republic of China

Tel/Fax +86 I87 88557524

Email beached@I26.com;

2697349858@qq.com
International Journal of Nanomedicine 2021:|6 297-3|4 
automatically controls the release of insulin by sensing stimuli produced by changes in the body's blood sugar concentration. $^{5-7}$ The glucose-sensitive drug delivery system can adjust the release of insulin according to the change in human blood glucose concentration. It not only stabilizes the blood sugar level at any time and improves the utilization rate of insulin, but also extends the time of drug administration and reduces the pain of patients with diabetes. ${ }^{8}$ Currently, there are three common glucose-sensitive drug systems, phenylboronic acid (PBA) and its derivatives, concanavalin A (Con A), and glucose oxidase (GOD). ${ }^{9}$ Con $A$ and glucose oxidase systems are proteins that exhibit volatile inactivation, poor stability, and high cost. In addition, Con A is biologically incompatible. The loss of Con A promotes mitosis, cytotoxicity, hepatotoxicity, teratogenicity, and other toxicities. ${ }^{10}$ On the other hand, PBA does not have these shortcomings. The glucose-sensitive polymer of PBA is a synthetic system that can be used for selfregulating and controlled release of drugs. Moreover, it is a common glucose-sensitive material because of its costefficiency and easy fabrication. ${ }^{11-14}$ This review focuses on the development process, performance, resolve resolution, and application of the glucose-sensitive polymer drug carrier of PBA and its derivatives, and also presents the prospective development direction and application in the field.

\section{Glucose-Sensitive Mechanism of PBA}

The controlled release of phenyl borate-based glucosesensitive materials to insulin and other drugs is effectuated via the contraction/expansion transition of phenyl borate or a competitive reaction.

\section{Contraction/Expansion Transition}

In aqueous solution, PBA has the hydrophobic form of the unionized planar triangle and the hydrophilic form of the ionized tetrahedron, and a dynamic equilibrium is detected between the two forms. Both forms bind specifically to substances containing o-diols, such as glucose, to form fiveor six-membered-ring phenyl borate complexes. The ionized form of PBA is combined with glucose in a reversible covalent bond to form a stable structure of phenyl borate (Figure 1). ${ }^{15}$ Therefore, due to the reversible effect of benzene, boric acid forms a copolymer hydrogel as a carrier of the insulin network, introduces the PBA group in the copolymer networks for glucose response, and takes charge of the PBA composite reaction, thereby causing the PBA dissociative deviation from the balance in the water and increasing the water charge density in the gel network. ${ }^{16}$ The joint effect of electrostatic repulsion and Donnan balance effect drives the PBA-bearing effect and insulin is released along with the rapid expansion of hydrogel. ${ }^{17}$

According to this characteristic of PBA, the drug carrier responds to the change in glucose concentration to release the loaded drug, and the higher the concentration of glucose, the faster the drug release rate, the greater the release amount.

\section{Competitive Response}

PBA smart materials also achieve the controlled release of drugs through competitive reactions. The complex of PBA and glycosylated drugs is placed in the glucose environment.

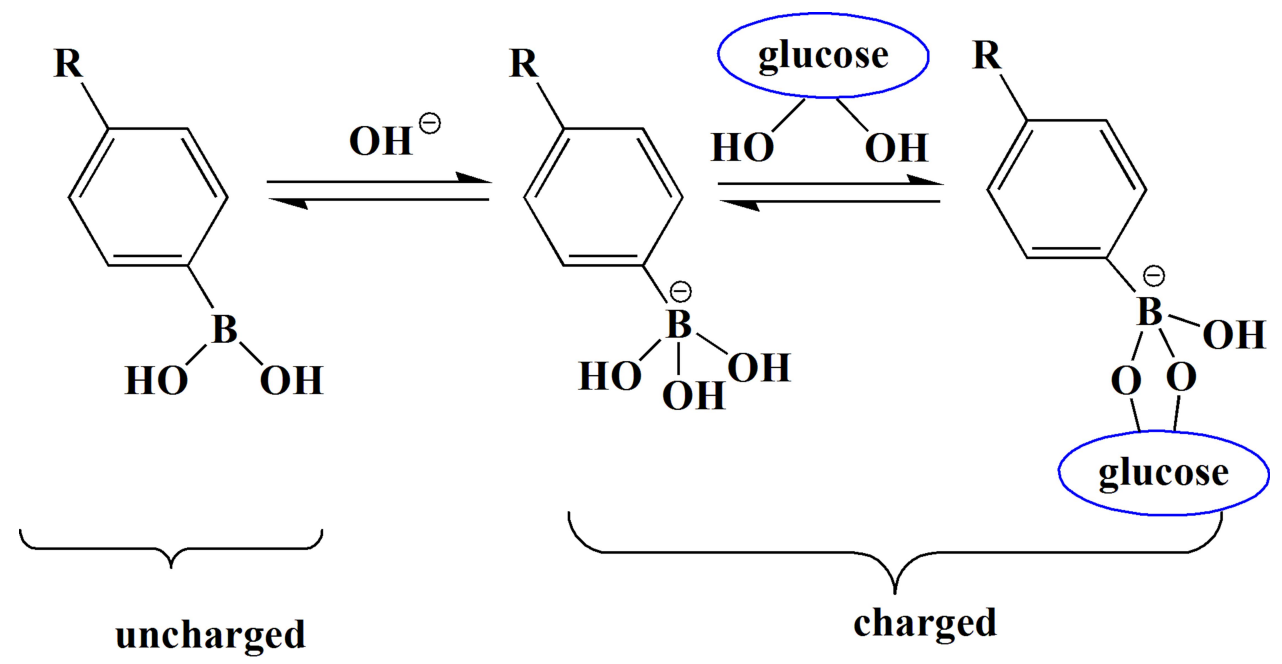

Figure I Schematic diagram of the interaction between PBA and glucose. 
The glucose reacts with the glycosylated drugs and replaces the drugs, such as insulin, to release the drug into the environment (Figure 2). ${ }^{18}$

This feature of specific binding to diols renders good glucose sensitivity to the functionalized carrier of PBA and its derivatives, which can be applied to the controlled release of insulin to construct a glucose-responsive selfregulating insulin delivery system. Furthermore, the PBAfunctionalized polymer carriers, such as hydrogel, microgel, microcapsule, and nanocarriers, have made significant progress. However, the application of PBA and its derivatives to the glucose-sensitive drug delivery system for diabetes therapy face many difficulties, such as the $\mathrm{pH}$ of human physiological environment is 7.4, while the PBA and its derivatives have higher $\mathrm{pKa}$ values (about 8-9). ${ }^{19,20}$ Under normal physiological environment, PBA polymer has a series of problems, such as poor water solubility and weak glucose response; the glucose response concentration is higher than the blood glucose level of DM patients.

The premise of PBA-based glucose-sensitive drug carrier used in diabetes therapy is to reduce its $\mathrm{pKa}$ value in order to realize the glucose-sensitive property under physiological $\mathrm{pH}^{21}$ and exert excellent glucose response in the human body's physiological environment. Various approaches have been used to resolve these issues, including the introduction of strong electron-withdrawing substituents in the benzene ring. This structural change leads to the electron-deficient boron atom of PBA, which makes it more acidic (low pKa). Matsumoto et al ${ }^{22}$ synthesized a PBA derivative 4(2-acrylamide-ethyl carbamoyl)-3-fluorophenylboronic acid (AmECFPBA), which harbors p-carbamoyl and M-fluoro substituents. The pKa of the new derivative was determined to be 7.2, which was significantly lower than the physiological $\mathrm{pH}$ value (7.4), thereby indicating that glucose sensitivity is present in physiological conditions. Watahiki et al ${ }^{23}$ made some modifications to the chemical structure of PBA and studied the effect of strong electron-withdrawing nitro group on glucose sensitivity. The dendrimer derivatives modified by 3-carboxyphenylboronic acid (3CPBA-D) and 3-carboxy5-nitrophenylboronic acid (3C5NPBA-D) were prepared, and the results showed that at $\mathrm{pH} 7.0,8.0$, and 9.0, the new PBA-based polymer dendrimers can be used in glucosetriggered release systems and are stable in glucose-free solutions at $\mathrm{pH}$ values 7.0-8.0. After introducing halogen into the meta position of PBA, Liu et $\mathrm{al}^{24}$ synthesized a new PBA derivative 2.4-difluoro-3-formyl -phenylboronic acid (DFFPBA), which has a low molecular weight for cis-diols and the binding $\mathrm{pH}$ is 6.0 . The monomers functionalized with DFFPBA are suitable for binding with glycol compounds in neutral and weakly acidic environments. Another method is to introduce an amino group into the PBA-containing polymer or at the ortho position of the PBA benzene ring and coordinate the lone pair of electrons of the amino nitrogen atom with the empty orbital of the boron atom, thereby reducing the $\mathrm{pKa}$ of the PBA-containing polymer. Wang

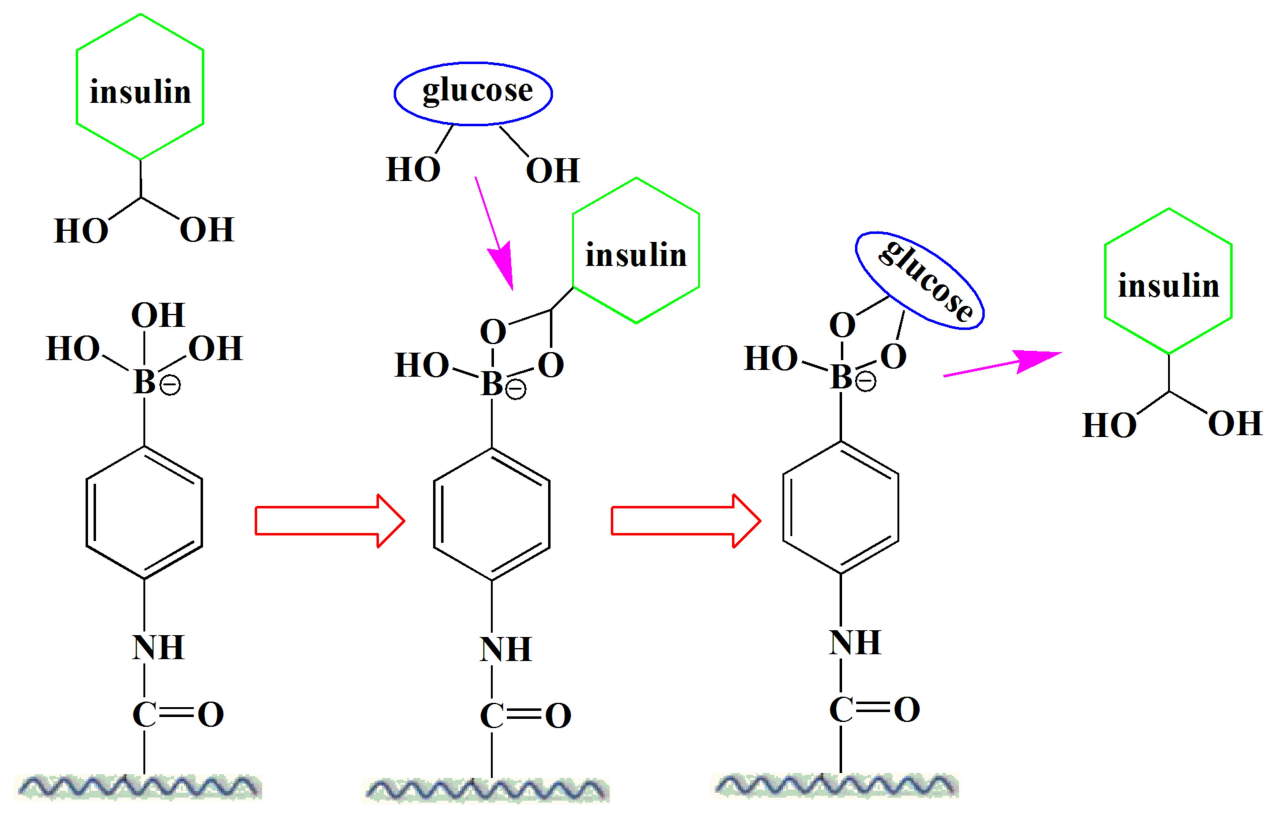

Figure 2 Schematic of release mechanism for glycosylated insulin from PBA-based systems upon glucose addition. 
et $\mathrm{al}^{25}$ introduced the amino groups in phenyl borate polymers, and the interaction between phenyl borate and amino groups improves the complexing ability and the response of glucose to physiological $\mathrm{pH}$. In addition, Akira et $\mathrm{al}^{22}$ studied the effects of different substituents on $\mathrm{pKa}$ of phenylboronic acid, they found the pKa of m-nitrobenzene boric acid and 3-fluoro-4-methoxybenzene boric acid derivative reached 7.8 and 7.4. Then they studied the glucose response behavior of the two phenylboronic acid derivative copolymer systems, the results showed that these copolymer systems produced a significant glucose sensitivity under physiological. Although the two methods mentioned above are effective in reducing the $\mathrm{pKa}$ of $\mathrm{PBA}$, and the self-regulated insulin release system based on these two methods has been widely studied, it also has the shortage of complex synthesis. Therefore, in recent years, researchers have tried to find new ways to reduce the pKa of PBA and its derivatives. One method is to use polymer materials as carriers and their characteristics to reduce the pKa value of PBA and its derivatives in glucose-sensitive drug delivery systems. ${ }^{26}$ The grafting or copolymerization of environment-sensitive monomers or polymers onto PBA and its derivatives support the PBA and its derivatives to adapt to the human environment, rendering it with high application prospect. Currently, there are few comprehensive and systematic introductions to the combination of PBA and environmentally sensitive monomers. Based on previous studies, researchers have found novel ways to make phenylborate-like glucosesensitive materials suitable for the human body environment and exert glucose response. According to different response factors, PBA-based glucose-sensitive drug carriers can be divided into $\mathrm{pH}$ response, temperature response, light response, enzyme response, and multifunctional response. Therefore, this study mainly reviews the research progress of environmentally sensitive carriers based on PBA in recent years, including carrier preparation methods, response to glucose, and controlled release of insulin and its application in diabetes.

\section{PBA and Environmentally Sensitive Carriers $\mathrm{pH}-$ Responsive PBA-Containing Biomaterials}

pH-sensitive polymers are composed of acidic or basic groups, which can accept or release protons in response to the changes in environmental $\mathrm{pH}$. Weak acids accept protons at low $\mathrm{pH}$ and release protons at neutral and high
pH. Most commonly used $\mathrm{pH}$-sensitive polymers are polyacrylic acid (PAAc), polymethacrylic acid (PMAAc), and chitosan. ${ }^{27,28}$ PAAc and PMAAc can be electrolyzed at specific $\mathrm{pH}$ with the change in environmental $\mathrm{pH}$. The rapid change in the net charge of the attached group alters the molecular structure of the polymer chain. Chitosan is a polycation that can be dissolved in an acidic solution. It is deprotonated with inorganic ions to deprotonate the first-order amino group and is separated at a $\mathrm{pH}$ close to that of neutral biopolymer. Moreover, the rich hydroxyl and amino groups in the chitosan chain can be used as reaction sites, which show strong affinity to phenylboronic acid. $^{21,29}$ Therefore, if PBA is modified into a $\mathrm{pH}$ responsive carrier, the synthesis of new polymers or crosslinking agents can lower the pKa value of PBA, thereby achieving glucose sensitivity under the physiological $\mathrm{pH}$ conditions of the human body. It has wide application prospect.

Ma et $\mathrm{al}^{30}$ modified PBA to the side groups of polyethylene glycol-b-polyacrylic acid (PEG-b-PAA) to obtain an amphiphilic block copolymer PEG-b-(PAA) containing PBA groups (PEG-b-PAA-co-PAAPBA), through the magic angle rotating nuclear magnetic study of boron. In the PAA -co-PAAPBA chain segment, the carboxylate has a certain stabilizing effect on the borate, which lowers the apparent pKa of the PBA group. These results showed an ideal glucose responsiveness (normal blood glucose concentration remains stable and rapid response under high blood glucose concentration) of the micelle, which is suitable for constructing an insulin delivery system.

Elshaarani et $\mathrm{al}^{16}$ synthesized poly(acrylamide -co -3-acrylamide -phenylboronic acid-chitosan grafted maleic acid) (p(AM-co-AAPBA-co-CSMA)s) hydrogel, which is used for glucose sensing and insulin release. The findings revealed that under physiological $\mathrm{pH}$ conditions, the hydrogel experienced two steps in response to glucose concentration; it contracted at low concentration and expanded at high concentration. Also, it has a high insulin load and encapsulation rate, and hence, can be used as an implantable glucose sensor and insulin release system.

Compared to single micelles, composite micelles have better stability and biocompatibility, which provides a novel idea for the construction of glucose-sensitive polymers. Gaballa et $\mathrm{al}^{31}$ used reversible addition-fragmentation chain transfer method (RAFT) to polymerize and modify the resulting poly(N-acryloyl morpholine-block-(Nacryloyl morpholine-copentafluoroacrylate) (p(AMP-b(AMP-co-PFPA))). After post-polymerization modification 
of PBA and diol-based block copolymer, p(AMP-b-(AMPco-PFPA)) copolymer reactive block was successfully synthesized in only two reaction steps (Figure 3). This polymer exhibited good glucose responsiveness under neutral $\mathrm{pH}$ conditions, and the composite micelles enhanced glucose responsiveness compared to pure PBA micelles under physiological conditions. In addition, the release of insulin from polymer micelles in glucose solution was also studied. The results showed that the composite micelles were stable under physiological conditions, while insulin release was enhanced at diabetic glucose concentration.

Another method to reduce the $\mathrm{pH}$ of PBA is by modifying polymers. Seno et $\mathrm{al}^{32}$ prepared a $\mathrm{pH}$ and glucose-sensitive multilayer film composed of PBA-modified polyallylamine hydrochloride (PAH) and polyvinyl alcohol (PVA), in which PBA-PAH and PVA are connected with each other through borate bond. This polymer film could be sensitive to human physiological glucose at $\mathrm{pH} 7.4$ but is stable in sugar-free solution (Figure 4), suggesting that is advantageous in constructing glucose induction and delivery system.

In recent years, mesoporous silica (MSN) has gained popularity in the fields of tissue engineering and drug delivery. ${ }^{33,34}$ It has excellent biocompatibility, adjustable mesopore size, large porosity, and strong modifiable properties. Liu et $\mathrm{al}^{35}$ used MSN to design a new type of glucose and pH dual-sensitive polymer-coated hybrid nanoparticles. PAA can be glycosylated with glucosamine to obtain P(AA-AGA). The polymer brush that blocks MSN pores is composed of $\mathrm{P}$ (AA-AGA) and 4.4-(ethylenedicarbamoyl)-phenylboronic acid (EPBA) and is formed by cross-linking of borate. The polymer brush covers the surface of MSN and acts as a "cover," that can embed the model drug insulin in the interior of MSN. When the concentration of glucose in the environment increases, the glucose molecules replace the sugar motifs on the polymer to compete with PBA, and this competitive combination opens the polymer brush of MSN and releases the embedded drug. When the $\mathrm{pH}$ value in the environment is lower than the acidity of pKa of EPBA, the specific binding between EPBA and polymer brush glycosyl is destroyed, and hence, the drug release behavior of MSN is affected by the $\mathrm{pH}$ value of solution (Figure 5).

In addition, the layer-by-layer (LbL) deposition technique based on hydrogen bond has $\mathrm{pH}-\mathrm{dependent} \mathrm{stability,} \mathrm{which}$ can be used to construct nano- and micro-components, and is suitable for oral insulin preparation and glucose-dependent insulin release system. ${ }^{36}$ The insulin-loaded microparticles can be prepared by depositing insulin on the surface of microparticles by $\mathrm{LbL}$ and forming polymers with opposite charges on the surface of the microparticles. ${ }^{37}$ Thus, LbL deposition is considered to be an ideal solution for preparing unstable protein drug delivery system, and this technology has been widely used in the preparation of protein films and microcapsules. Incorporating PBA into LbL module containing insulin can be developed into bioartificial pancreas, which makes great contribution to the further development of patientfriendly insulin delivery system. For example, Sato et $\mathrm{al}^{38}$ prepared a glucose-reactive multilayer capsule by LbL deposition of degradable polymers, such as alginate derivatives and polyvinylpyrrolidone (PVPON), according to the number of hydrogen bonds. This hydrogen-bonded LbL multilayer film solves the problem of poor stability under physiological conditions and that insulin is decomposed by proteolytic enzymes under acidic conditions (such as in the gastric environment), which leads to insulin instability during transportation. Also, the glucose response in the human body and the bioavailability of oral insulin preparation are improved.

The above studies have shown that compared to unmodified PBA, pH-sensitive polymers based on PBA have
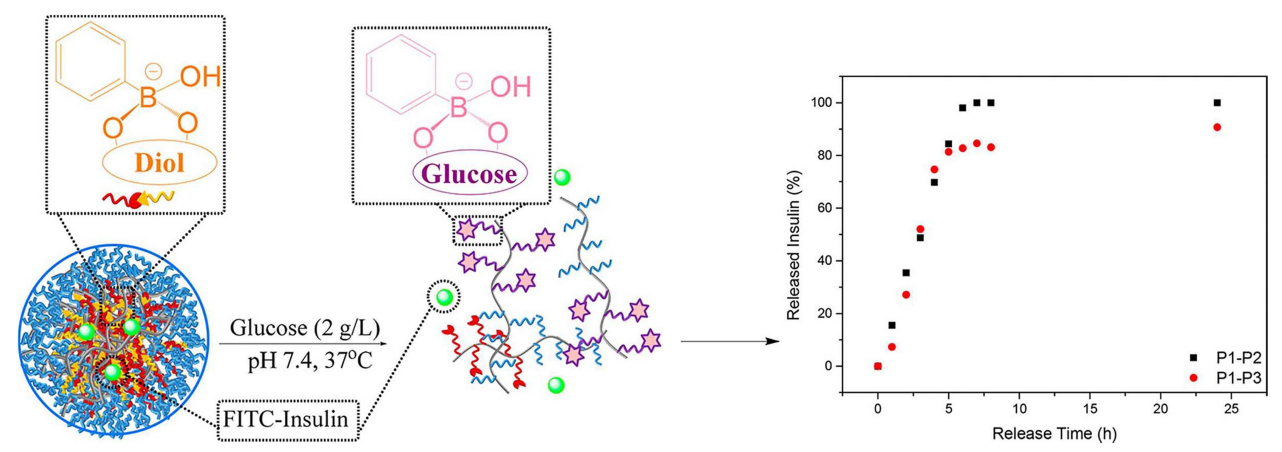

Figure 3 Schematic illustration of the formation of complex micelles of poly[(AMP)-b-(AMP-co-PBA)]-PI and Poly[(AMP)-b-(AMP-co-GA)]-P2 or Poly[(AMP-b-poly(AMPco-PRD)]-P3. Self-assembly of PI was investigated at a-I concentration of I mg/mL at pH 7.4 below its pKa, at which the PBA exists in the uncharged/hydrophobic form. Notes: Reprinted from Gaballa H, Theato P. Glucose-Responsive Polymeric Micelles via Boronic Acid-Diol Complexation for Insulin Delivery at Neutral pH. Biomacromolecules. 2019;20(2):87I-88I. Copyright (C) (2019), with permission from American Chemical Society. ${ }^{31}$ 

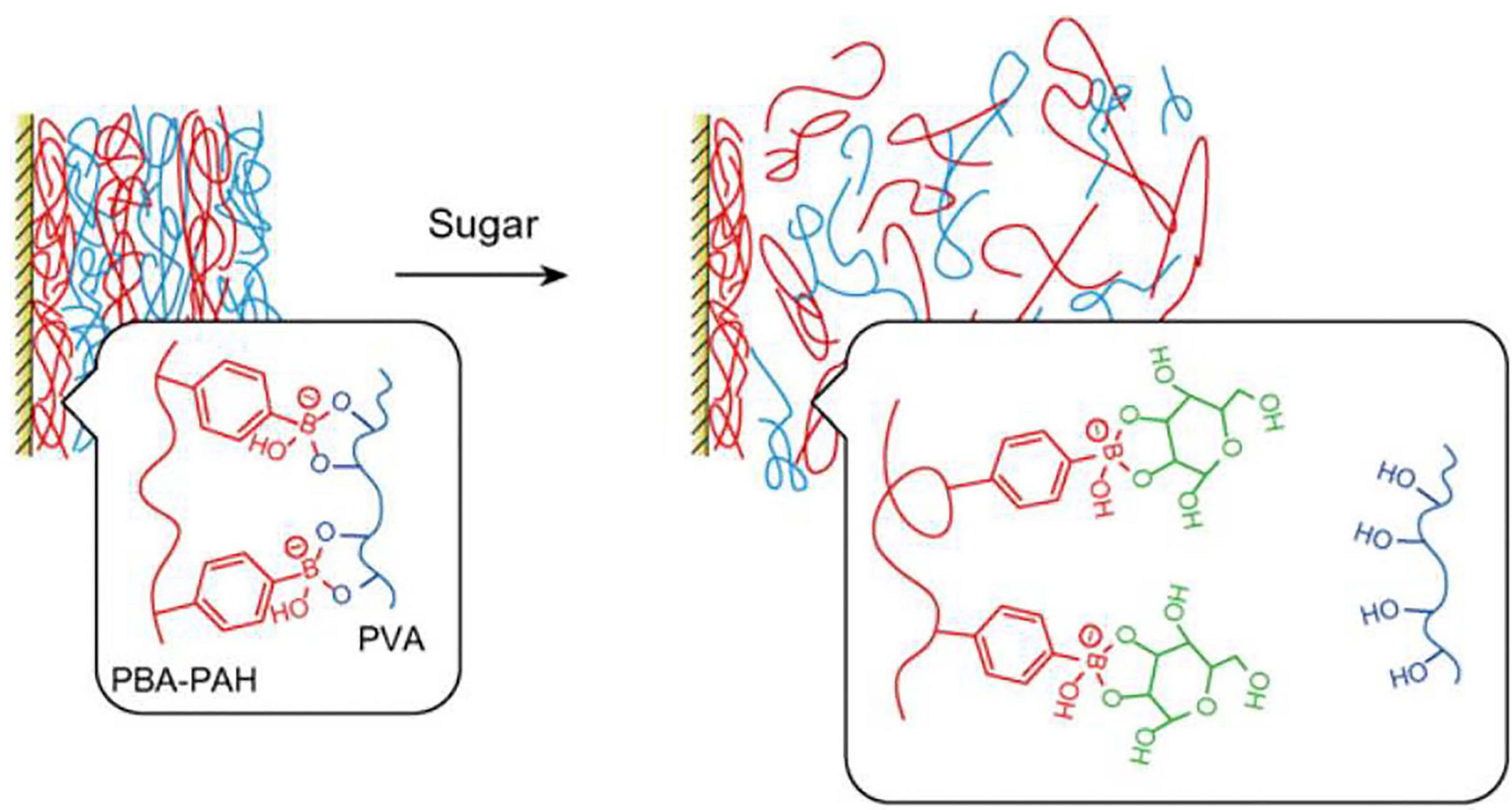

Figure 4 A schematic illustration of the mechanism for sugar-induced decomposition of (PBA-PAHPVA) 10 films. Sugars competitively bind to PBA-PAH in the multilayer films to replace PVA because sugars contain I.2- and I.3-diol moieties, resulting in destabilization or decomposition of the films.

Notes: Reprinted from Seno et al. $\mathrm{pH}$ - and sugar-sensitive multilayer films composed of phenylboronic acid (PBA)-modified poly(allylamine hydrochloride) (PBA-PAH) and poly(vinyl alcohol) (PVA): A significant effect of PBA content on the film stability. Materials Science and Engineering: C, 2016;62:474-479. Copyright (C) (20I6), with the permission from Elsevier. ${ }^{32}$
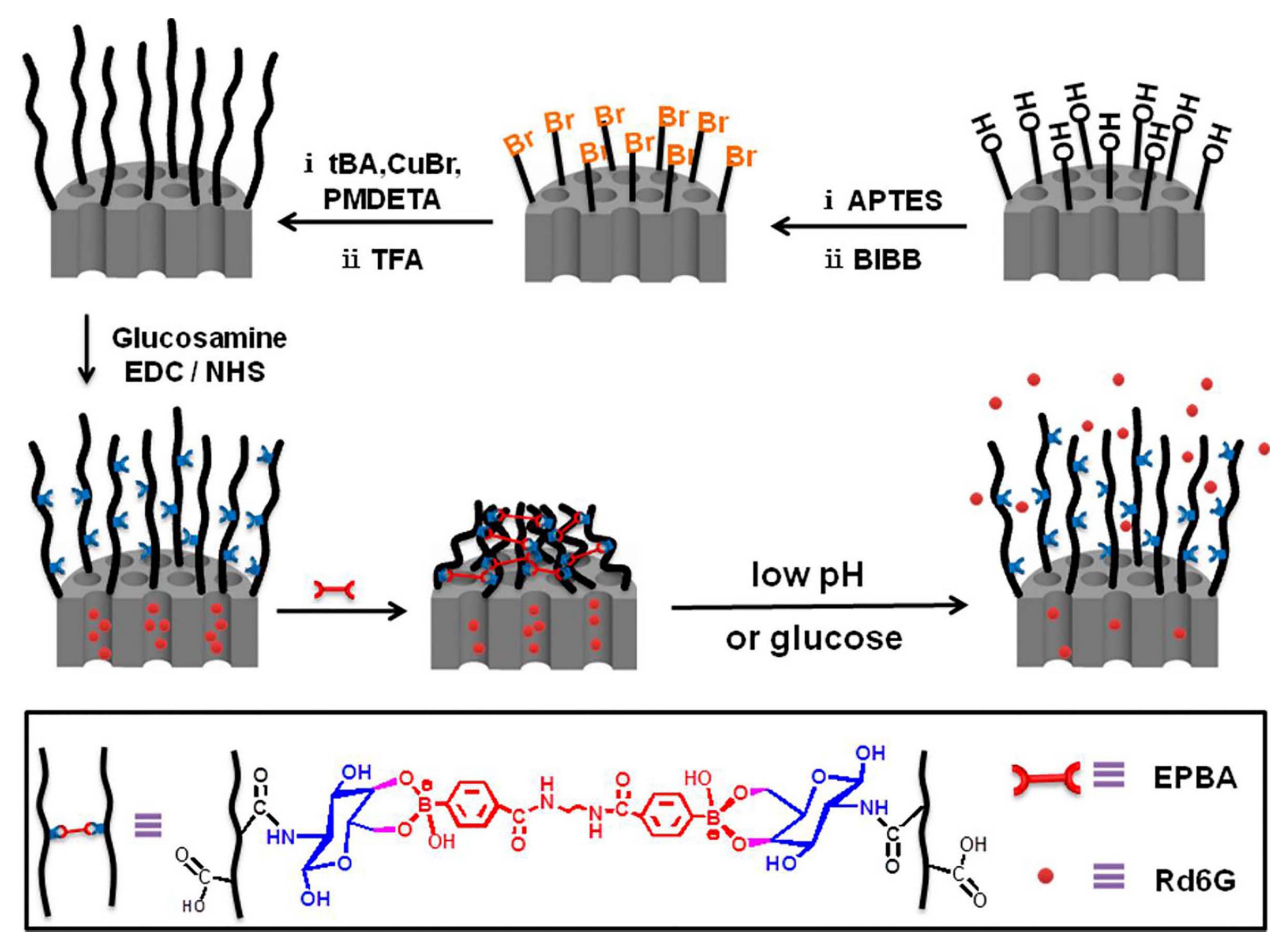

Figure 5 Cumulative release of Rd6G from MSN-PAA-AGA in PBS ( $\mathrm{pH}=7.4)$ with different concentrations of glucose; the combination of two stimuli exhibited an obvious enhanced release capacity.

Notes: Reprinted from Tan L, Yang MY, Wu HX et al. Glucose- and pH-responsive nanogated ensemble based on polymeric network capped mesoporous silica. ACS Appl Mater Interfaces. 2015;7(II):6310-6316. Copyright (C) (2015), with the permission from the American Chemical Society. ${ }^{35}$ 
better glucose responsiveness and self-controlled insulin release under human physiological $\mathrm{pH}$ conditions, which highlights the application of $\mathrm{pH}$-sensitive polymers in controlling blood glucose changes under physiological condition to treat diabetes.

\section{Temperature-Responsive PBA-Containing Biomaterials}

The solubility of temperature-sensitive polymers could change with altered temperature, and water-based thermosensitive polymer solutions exhibit temperature-dependent and reversible sol-gel transitions under appropriate conditions. This transformation can control the release rate of the combined drug and maintain physical and chemical stability. The most commonly used temperature-sensitive polymers include poly(N-isopropyl acrylamide) (PNIPAM), poly (N-vinyl caprolactam) (PNVCL), and phosphazene derivatives. $^{39,40}$ Therefore, if PBA is polymerized with a temperature-responsive polymer, the overall structure and surface properties of the polymer can be changed by the temperature under the physiological conditions. The temperature-sensitive polymer is used as a temperature-responsive actuator, and PBA is used as a glucose-responsive sensor; both couplings can ascribe dual-response characteristics of temperature and glucose to the final product, ${ }^{41}$ which expand the scope of their respective applications. Wu et $\mathrm{al}^{42}$ used the temperature response characteristics of 3-acrylamide and used 2.2-azobisisobutyronitrile (AIBN) as the initiator, n-vinyl caprolactam (NVCL), and 3-acrylamidoboronic acid (AAPBA) prepared poly-n-vinylcaprolactam-copolymeracrylamide boronic acid p(NVCL-co-AAPBA). These new nanoparticles are monodispersed submicron particles, sensitive to glucose and temperature (Figure 6). The nanoparticles have good insulin loading characteristics, do not affect the conformation of insulin, and show low toxicity to cells and animals.

PNIPAm is the most commonly used polymer to construct temperature-sensitive nanoparticles. The low critical solution temperature (LCST) of PNIPAm can be adjusted by copolymerization with hydrophilic or hydrophobic monomers. Li et $\mathrm{al}^{43}$ synthesized copolymers containing $\mathrm{N}$-isopropyl acrylamide (NIPAAm) and AAPBA by free radical polymerization and compared the response behaviors of the copolymers under varied concentrations of glucose. The results showed that the introduction of NIPAAm makes the composite material thermally sensitive, and the addition of AAPBA reduces the molecular weight of the copolymer, enhances the intermolecular aggregation of nanoparticles, and reduces the LCST of the composite material such that the copolymer reacts to glucose. Aslam et $\mathrm{al}^{44}$ used MBA as a crosslinking agent to synthesize glucose-sensitive 3-acrylamidoboronic acid (APBA) and temperature-sensitive P(NIPAm-AAc) shell nanogel particles through free radical polymerization. The synthesized particles are approximately $100 \mathrm{~nm}$ in size and crosslinked with each other; the synthesized colloidal nanogel particles have thermal response characteristics. As the temperature of the external environment increases or decreases, the size of the particles dispersed in the aqueous solution also changes. The sensitivity of these nanogel particles to temperature and glucose suggested deemed them usable for self-regulating insulin delivery.

In addition to the above-mentioned temperaturesensitive carriers in the form of micelles and nanometers, temperature-sensitive carriers in the form of vesicles have also attracted the attention of researchers due to their advantages. Vesicles are highly hydrophobic, which is beneficial to increasing the loading of drugs, and the double-layer membrane structure of vesicles is critical for maintaining the biological activity of drugs. Yang et $\mathrm{al}^{45}$ used PBA-containing temperature-sensitive block copolymer PNIPAM-bP (Aspco-AspPBA) to selfassemble into core-shell (CS) micelles with PNIPAM core and $\mathrm{P}($ Asp-co-AspPBA) shell, with LCST higher than that of PNIPAM. After adding glucosamine (GA) to block copolymer PEG-bP (Asp-co-AspGA), due to the cross-linking between PBA- and GA-blocks, a core-shellcrown (CSC) composite glue is formed in the bundle. Then, the CSC composite micelles are stored below the LCST of PNIPAM, and the expanded PNIPAM core, cross-linked $\mathrm{P}($ Asp-co-AspPBA)/P(Asp-co-AspGA) vesicle membranes, and PEG electrodes are acquired. The halo of polymer vesicles has a hydrophilic membrane, which is conducive to the penetration of prepared substances at a physiological $\mathrm{pH}$ of 7.4, and FITC insulin was used as a model protein. After encapsulation in polymer vesicles, the effect of insulin release resulted in successful drug release triggered by both continuous and on-off glucose.

Liu et $\mathrm{al}^{46}$ used the temperature response characteristics of poly isopropyl acrylamide (PNIPAM) to prepare polyethylene glycol-block-poly(aspartic acid-co-asparaginyl phenylboronic acid) (PEG-bP (Asp-co-Asp PBA)) and poly isopropyl acrylamide-block-poly (aspartic acid-co- 


\section{A without insulin}

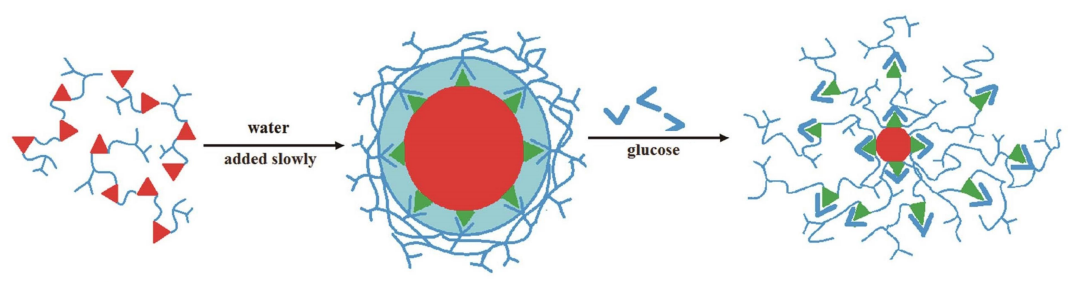

B insulin-loading

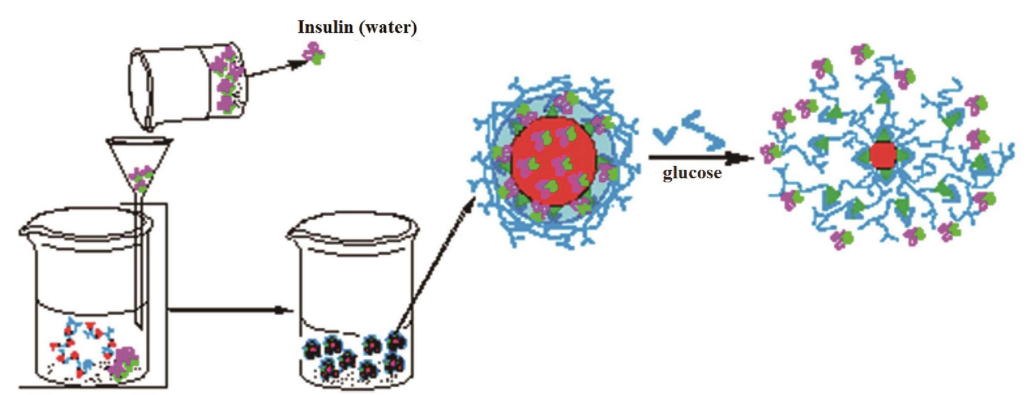

NVCL(hydrophilic)
uncharged AAPBA(hydrophobic)
charged AAPBA(hydrophobic)

Figure 6 Schematic representation of temperature- and glucose-sensitive $\mathrm{p}(\mathrm{N}$-vinylcaprolactam -co-acrylamidophenylboronic acid) $\mathrm{p}(\mathrm{NVCL}-\mathrm{co}-\mathrm{AAPBA})$ nanoparticles. $(\mathbf{A})$ It is a schematic diagram of nanoparticles without insulin; (B) It is a simple schematic diagram of the nanoparticle production process after adding insulin.

Notes: Reprinted from Wu JZ, Bremner DH, Zhu LM. Synthesis and evaluation of temperature- and glucose-sensitive nanoparticles based on phenylboronic acid and $\mathrm{N}$-vinylcaprolactam for insulin delivery. Mater Sci Eng C Mater Biol Appl. 2016; 69:1026-1035, with permission from Elsevier. ${ }^{42}$

asparaginyl phenylboronic acid) (PNIPAM-bP (Asp-coAsp PBA)) to block the copolymerization. At $37^{\circ} \mathrm{C}$, the temperature-sensitive PNIPAM layer collapses on the surface of the micelle core and forms a continuous film structure. The PEG layer is sandwiched between the polymer micelle core and the PNIPAM film layer. The composite micelle changes from the classic "core-shell" structure to the special "core-shell-crown" structure (Figure 7). The composite micelle has a stable structure and exhibits a reversible glucose-responsive swelling behavior. Importantly, it has the characteristics of a repeated "onoff" release of insulin.

The main advantages of thermosensitive polymers based on PBA are that toxic organic solvents are avoided, side effects in the human body are reduced, hydrophilic and lipophilic drugs (such as insulin) can be released simultaneously, and the characteristics of drug delivery at specific sites are exhibited, which is advantageous in insulin drug delivery in diabetes. Despite these characteristics, the thermosensitive polymer system based on PBA exhibits disadvantages of high drug release and short drug release time, which need to be investigated further.

\section{PBA Biomaterials Containing Long-Acting Sustained-Release Polymers}

Due to the short release time of the PBA drug delivery system, the control of the blood sugar of diabetic patients cannot achieve the effect of long-term regulation of blood sugar stability, which easily leads to fluctuations in blood concentration and adverse reactions. Long-acting sustained-release formulation (SRP) delays the release rate of the drug from the dosage form and reduces the absorption rate of the drug into the body, thereby enhancing the therapeutic effect. ${ }^{47}$ The long-acting sustained-release agents are currently research hotspots due to the advantages, such as prolonging the activity of drugs, reducing the frequency of medication, and improving patient compliance. $^{48,49}$ Since most PBA nanoparticles are stable even after the release of the drug, if insulin is embedded or adsorbed on the surface of the polymer molecule of the long-acting sustained-release agent, the glucose sensitivity and the long-acting drug are combined, in order to slow the release of the drugs. Such materials include methacrylate (PMMA), polylactic acid (PLA), polylactic 


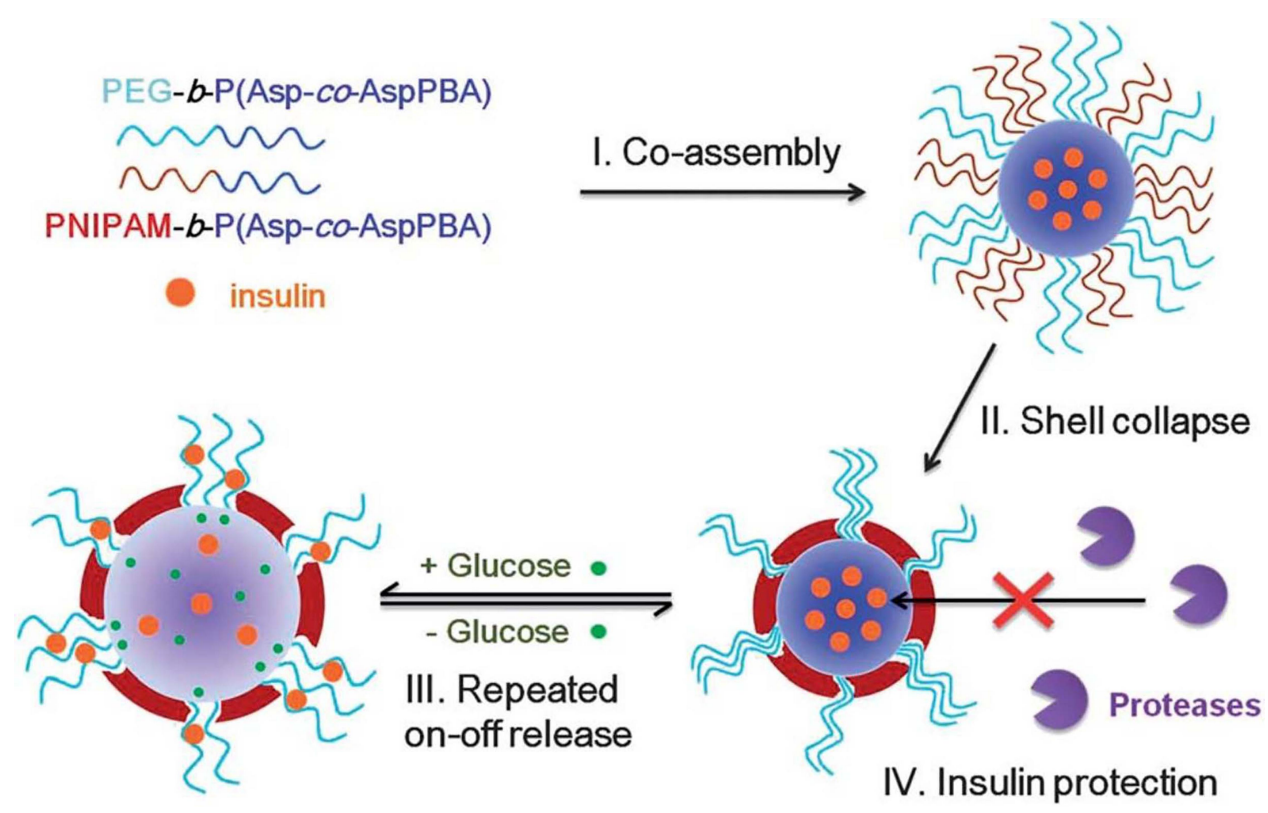

Figure 7 Schematic illustration of the glucose-responsive complex polymeric micelle (CPM) with effective glucose responsiveness and reversible swelling for repeated "onoff" release and insulin protection under physiological conditions.

Notes: Reprinted from Liu G, Ma R, Ren J et al. A Glucose-Responsive Complex Polymeric Micelle Enabling Repeated On-Off Release and Insulin Protection. Soft Matter. 2013, 9 (5):1636-1644. Copyright (C) (2013), with the permission from The Royal Society of Chemistry. ${ }^{46}$

acid glycolic acid (PLGA), and cyclodextrin (CyD) ${ }^{50}$ Zhu et $\mathrm{al}^{51}$ formulated PLGA, polyvinyl acetate (PVA), and $\mathrm{p}$ (AAPBA-co-NVCL) into microspheres by LbL selfassembly technology. The long-acting glucose-sensitive porous microspheres are long-acting, slow the release of insulin, and reduce the glucose of rats for 15 days. At the same time, when releasing insulin, the drug release rate is elevated according to the glucose concentration in the blood.

In the study by Abu et $\mathrm{al}^{52}$ glycol/ $\alpha$-cyclodextrin $(\alpha$ $\mathrm{CyD}$ ) and $\gamma$-CyD polypseudor-otaxane (PPRX) hydrogels contained high molecular weight polyethylene glycol (PEG). In addition, $\alpha / \mathrm{CyD}$ PPRX with $\alpha$-CyD and $\gamma$ CyDPPRX hydrogels were formed by inserting one PEG chain into the $\alpha$-CyD cavity and two PEG chains into the $\gamma$ CyD cavity. In vitro release studies have shown that the dissolved release of CyDs PPRX hydrogel follows the order of $\gamma$-CyDPPRX hydrogel $>\alpha$-CyDPPRX hydrogel. In animal experiments, after subcutaneous injection of $\gamma$-CyDPPRX hydrogel to rats, the serum insulin level was significantly prolonged, while the area under the serum concentrationtime curve increased, which reflected its prolonged bloodsugar-lowering effect. In summary, these results indicated that $\gamma$-CyDPPRX hydrogel can be used as a sustainedrelease system for insulin injection. If it is used in combination with PBA, it can extend the drug release time and increase the sensitivity to glucose, such that smart and longterm drug release can be achieved.

Previous studies have shown that compared to glucose, the binding constant of $\mathrm{N}$-acetylneuraminic acid (Neu5Ac) of PBA and sialic acid is 7.4-fold higher than that of glucose $(5.1 \mathrm{M}-) .{ }^{53}$ However, Neu5Ac exists at the end of sugar chains on the cell surface, which renders cell adhesion property to PBA, such that the PBA-modified polymers show an affinity for cells. ${ }^{54-58}$ Thus, researchers have developed a new long-acting drug concept based on the affinity of PBA to cell surface sugar chains. Using PBA to modify the cell adhesion of the drug prolongs the activity of the drug, because the PBA-modified drug may escape degradation and excretion, resulting in slow and long-lasting activity. Ohno et al ${ }^{59}$ prepared PBA-modified insulin (PBA-Ins) and injected it intravenously into diabetic rats to evaluate the duration of their hypoglycemic activity; also, the study evaluated the difference between intravenous and subcutaneous injections. The results showed that PBA-Ins had longer hypoglycemic activity than natural insulin, as observed by monitoring the blood glucose levels. The expected interaction between the PBA portion of the cell surface in the subcutaneous tissue and blood vessels and the sugar chain is shown in Figure 8. Unlike intravenous PBA-Ins, subcutaneous PBA-Ins shows low glucose metabolic activity. 
Compared to traditional preparations, sustained-release microsphere preparations reduce the number of administrations, improve patient compliance, reduce side effects, and improve curative effects. In recent years, these microspheres have become a hotspot in the development of new drug formulations. However, the drug release cycle of this formulation is long, and hence, it is necessary to establish an in vitro release acceleration test method to investigate the in vitro release behavior of the long-acting microspheres. How to choose the appropriate acceleration conditions to indicate the long-term release behavior of the microspheres is also a critical aspect of ball research.

\section{Light-Responsive PBA-Containing Biomaterials}

The main advantages of photopolymers are water solubility, biocompatibility, and biodegradability. Light stimulation can be applied immediately, which enables them to release sol-gel stimuli instantly. This makes the photoresponsive polymers suitable for various engineering and biomedical applications. Such polymer can control the release of drugs in space and time, thereby conducive in drug release application. The light-responsive carrier can use the light of a specific wavelength as a stimulus, and the drug encapsulated in the light-responsive polymer is released or activated after irradiation by a light source from outside the body. ${ }^{60}$ Therefore, the introduction of PBA group-containing molecules and/or polymers into the photosensitive polymer can form a glucose-sensitive drug release system with adequate biocompatibility, rapid response, and glucose responsiveness after injection into the human body.

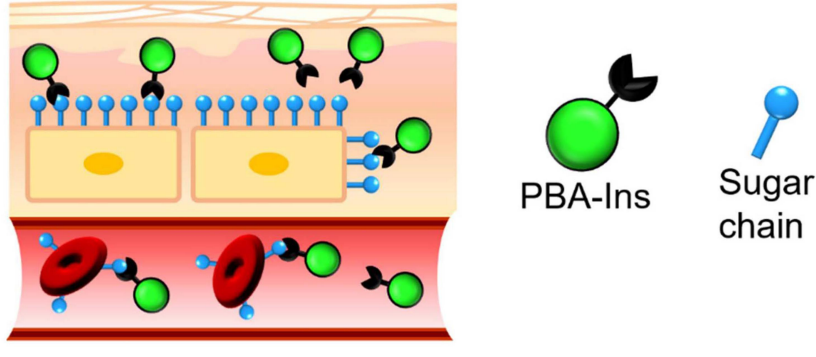

Figure 8 Expected interactions between PBA-modified insulin (PBA-Ins) and sugar chains on the cell surfaces in subcutaneous tissue and blood vessel. The cell adhesiveness of the PBA-modified drug prolongs the drug activity because the cellattached PBA-modified drug may escape from degradation and excretion, producing slow and long-lasting activity.

Notes: Reprinted with permission from Pharmaceuticals. Ohno Y, Kawakami M, Seki T et al Cell Adhesive Character of Phenylboronic Acid-Modified Insulin and Its Potential as Long-Acting Insulin. Pharmaceuticals (Basel). 2019;12(3):121. ${ }^{59}$
The photo-fragmentation type PBA-functionalized polymer material represented by nitrobenzene derivative materials undergoes phase change behavior under ultraviolet light and can be used in glucose-sensitive drug delivery systems. Jiang et $\mathrm{al}^{61}$ adopted the experimental design and used RAFT polymerization method to use poly(methoxy polyethylene glycol acrylamide) (Me PEGA) as the initiator of the copolymerization between AAPBA and o-nitrobenzyl acrylate (NBA). The synthesized polymer micelles are sensitive to UV light and glucose and can be used for insulin encapsulation. Data show that under visible light irradiation, the cumulative release of insulin within $30 \mathrm{~min}$ is only $20 \%$, while under ultraviolet light $(\lambda \max =365 \mathrm{~nm}$, $75 \mathrm{~mW} / \mathrm{cm}^{2}$ ), the total amount of insulin released within $30 \mathrm{~min}$ is less up to $98 \%$. In addition, the polymer also has the controllable release properties of glucose-responsive insulin. Based on the specific binding of PBA and polyols, the cumulative release of insulin in a solution with 25 $\mathrm{mmol} / \mathrm{L}$ glucose concentration is 18 -fold that of a solution without glucose.

As a light-responsive carrier, cellulose also has excellent biocompatibility and mechanical properties as well as unique characteristics, such as hydrophilicity, optical activity, and biological functionality. Using cellulose as a matrix, the introduction of PBA group-containing molecules and/or polymers can form a new type of biohydrogen with good biocompatibility and glucose responsiveness, which has broad application prospects in the field of biomedicine. ${ }^{62}$ Peng et al ${ }^{63}$ used cellulose as raw material, molecules, and/or polymers containing PBA groups, and successfully prepared a new type of smart cellulose/ 4-ethylene with glucose and $\mathrm{pH}$ responsiveness through internal electron beam superimposition technology. Under the overlapped electron beam irradiation, the carbonyl group in the polymerization of 4-toluene benzoic acid underwent a grafting and crosslinking reaction, forming a double-crosslinked network (VPBA) composite biological hydrogel in the composite hydrogel. The glucose response characteristics and the self-regulation of release and release of this composite hydrogel were analyzed, and it was found that the combination of cellulose and VPBA can acquire a satisfactory $\mathrm{pH}$ value, glucose responsiveness, and self-regulated drug release.

Reduced graphene oxide (rGO) is a photothermal agent that absorbs near-infrared light (NIR) with the advantages of high spatial and temporal resolution and a high degree of benefit in drug delivery. ${ }^{64-67}$ Compared to other allotropic carbons, reduced graphene oxide has the advantage 
that under low-power near-infrared irradiation, reduced graphene oxide can also effectuate rapid photothermal conversion. This makes reduced graphene oxide the preferred filler for polymer copolymers and composite hydrogels. ${ }^{6-70}$ In addition, reduced graphene oxide has a high surface area and strong fluorescence quenching, making it the most promising tool for biomolecule detection applications. Polymerizing reduced graphene oxide with PBA and its derivatives can render glucose- and lightresponsiveness to the glucose, and the reduced graphene oxide functionalized with phenylboronic acid can be used as a sensor for glucose sensing and detection of the glucose molecule in the human body. ${ }^{71}$ For example, Basiruddin et $\mathrm{al}^{72}$ used PBA-functionalized reduced graphene oxide (rCO-PBA) and glycol-modified fluorescent probes to synthesize a fluorescence-based nanosensor for the detection of biologically important glucose molecules. The results showed that the PBA-functionalized reduced graphene oxide nanosensor can detect glucose molecules in $2-75 \mathrm{mg} / \mathrm{mL}$ aqueous solution. Teodorescu et $\mathrm{al}^{73}$ used reduced graphene oxide impregnated polyethylene glycol dimethacrylate hydrogel (PEGDMA-rGO) formula to load them effectively with insulin. The PEGDMA-rGO hydrogel heated by light-induced heating in the NIR allows efficient insulin release. The experimental results confirmed that the near-infrared irradiated hydrogel does not affect the biological and metabolic activities of the released insulin.

Thus, it is possible to combine the two and use continuous blood glucose monitoring (CGM) device to measure the interstitial glucose concentration in the tissue together with the insulin release pump and form an automatic feedback loop that can deliver insulin in the event of hyperglycemia, and vice versa suspend insulin delivery when hypoglycemia occurs. In the future, researchers can expand in this area and use phytopharmacology to achieve remote control of glucose homeostasis in the body.

\section{Glycolipid-Combined PBA-Containing Biomaterials}

Improving the safety of drug production is crucial to ensure healthy aging of the human body. In recent years, functional biomaterials have been widely studied in the drug delivery system because of their low price and good biocompatibility in human body, and have been found to have great application prospects. ${ }^{12,74-76}$ Sugar polymer is a functional polymer material synthesized by different biological reactions and chemical reactions. It not only retains the special properties of sugar, but also has new functions such as good hydrophilicity, biocompatibility, and biodegradability. Therefore, if the carbohydrate polymer is introduced into the polymer based on phenylboronic acid (with abundant hydroxyl groups), the sugar-based part of the polymer will be replaced by glucose in the external environment, which will destroy the cyclic borate structure, promote the glucose responsiveness of the polymer, and improve the hydrophilicity and biocompatibility of phenylboronic acid. For chronic diseases (such as type II diabetes), it maintains normal blood glucose homeostasis for a long time with minimal side effects.

Guo et $\mathrm{al}^{77}$ synthesized an amphiphilic block copolymer based on PBA and carbohydrate polymer (3-acrylamidephenylboronic acid-b-2 acrylic pyranose) p(AAPBAb-AGA) p (PBA-b-AGA). In addition, insulin as a model drug can be encapsulated in copolymer nanogels, with drug loading capacity of up to $8.2 \%$. With increasing glucose concentration, the drug release also increases and exhibits high glucose-responsiveness. The introduction of sugar carbohydrates improves the biocompatibility of PBA. However, the nano polymer has the shortcoming that the cumulative release time is only $16 \mathrm{~h}$.

Previous studies demonstrated that galactose and glucose react with dienedioic acid to form compounds containing a single enoic acid, which can be polymerized to form sugar polymers. This copolymer responds to glucose levels in the blood and further increases the cumulative release time. Therefore, Wu et $\mathrm{al}^{78}$ used AAPBA and 6-O-vinylzeloyl-D galactose (OVZG) as raw materials, S-1-dodecyl-S-( $\alpha^{\prime}, \alpha^{\prime}$ Dimethyl- $\alpha "$-acetic acid) trithiocarbonate (DDATC) as chain transfer agent, 2-azobisisobutyronitrile (AIBN) as initiator, and dimethyl formamide (DMF) as the solvent. Poly3acrylamidophenylboronic acid-b-6-O-vinyl-D-galactose nanoparticles ( $\mathrm{p}(\mathrm{AAPBAb}-\mathrm{OVZG})$ ) were synthesized by a twostep method (Figure 9). When using insulin as a model drug, these nanoparticles had high encapsulation and drug loading rates. At high glucose levels, insulin release is also increased. Moreover, nanoparticles have shown low toxicity in cell and animal studies, and can effectively reduce blood glucose levels in mice for $>96 \mathrm{~h}$. Zhong et al, ${ }^{14}$ also successfully synthesized the galactosyl block copolymer 6-O-sulfuric acid sebacic acidD-galactopyran ester tert-butyl 3-acrylamide-phenylboronic acid ( $\mathrm{p}(\mathrm{OVNG}-\mathrm{b}-\mathrm{AAPBA}))$. After encapsulating the nanoparticles with insulin, the increased AAPBA in the polymer, the drug loading, and high encapsulation efficiency can effectively maintain the 96-h blood glucose level of diabetic mice. The 


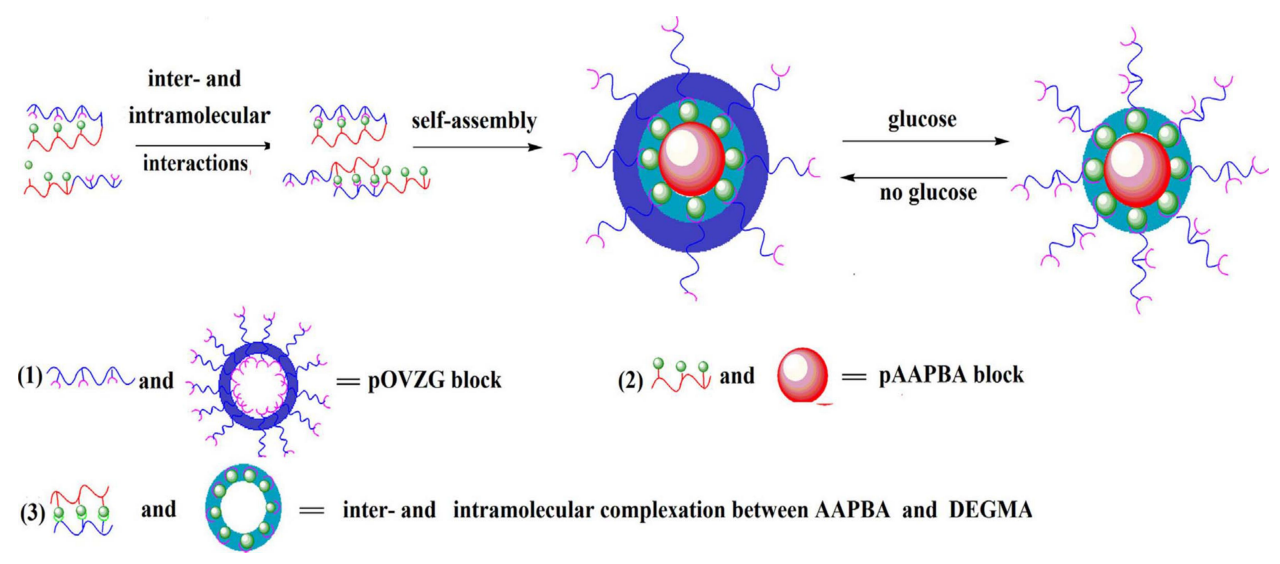

Figure 9 Schematic diagram of the reaction mechanism of $P(A A P B A-b-O V Z G)$ nanoparticles to glucose. ${ }^{78}$

Notes: (I) OVZG was synthesized by chemoenzymatic method, and pOVZG block was formed by self-polymerization. (2) Under the influence of intermolecular interaction, AAPBA self-assembly forms pAAPBA block. (3) AAPBA and DEGMA interact under intermolecular and intramolecular complexation. Reproduced from Wu JZ, Bremner DH, Li HY, et al. Phenylboronic acid-diol crosslinked6-O -vinylazeloyl-d-galactose nanocarriers for insulindelivery. Mater Sci Eng C Mater Biol Appl. 20I7;76:845-855. ${ }^{78}$

above experimental studies showed that the insulin-loaded galactosyl polymer-3-acrylamide -phenylboronic acid nanoparticles have good sustained-release/glucose-responsive drug release characteristics and can play an active role in the treatment of diabetes.

In addition to the subcutaneous injection method described above, ${ }^{79}$ the phenylbutyrate glycosylated polymer nanoparticles have the characteristics of targeting, prolonged blood circulation time, high drug loading rate, and biocompatibility. The nasal drug delivery system has also been actively studied by many researchers for its ability to prevent the degradation of proteins and peptides in the gastrointestinal tract and the metabolism of liver enzymes; this approach is also a promising route for drug delivery of proteins and peptides. Zheng et $\mathrm{al}^{75}$ prepared an amphiphilic phenyl borate-based carbohydrate polymer (2-lactose-imide-ethylmethacrylate-random-3-acrylamide phenylboronic acid) (p(LAMA-r-AAPBA)), which was then assembled into nanoparticles that effectively encapsulated insulin as a model drug. The results suggested that the nanoparticles contained up to $12 \%$ of the drug, and the release of insulin could be controlled by changing the composition of glycan polymers, which in turn, significantly reduced the blood glucose level of diabetic rats. The cell viability test also showed that the nanoparticles of $p$ (LAMA-r-AAPBA) had good cell compatibility. Wei et al${ }^{80}$ developed boric acid-modified dextran as a vehicle for basal insulin delivery. In diabetic rats, the treatment of the obtained nanoparticles enhanced insulin absorption in the nasal cavity and prolonged their residence time in the nasal cavity.
However, since it is difficult to control the dose in nasal administration, the problem of uneven distribution of drugs in the nasal cavity needs to be investigated further.

\section{Amino Acids-Combined PBA-Containing Biomaterials}

Biocompatible and biodegradable amino acids have been widely used in various biomedical applications, such as tissue engineering, ${ }^{81}$ medicine, and gene delivery. ${ }^{82,83}$ Studies have found that peptides synthesized by amino acids can be used as functionalized materials for PBA in the glucose-responsive insulin delivery system. ${ }^{84}$

Zhao et $\mathrm{al}^{85}$ prepared novel nanogels by ring-opening polymerization of-benzyl-l-glutamic acid n-carboxylic anhydride and-properly-glutamic acid n-carboxylic acid anhydride (PLG NCA) with amino-methoxy polyethylene glycol as initiator on azide-modified sugars to the PLG unit and crosslink them with aliphatic acylaminophenylboronic acid (AAPBA). The model drug insulin is loaded into a glucose-sensitive polypeptide nanogel where the presence of glucose triggers the release. Moreover, in vitro tetrazolium and hemolysis tests have shown that these nanogels have biocompatibility properties, and the smart nanogels are promising for drug self-regulation.

Li et $\mathrm{al}^{86}$ designed new insulin encapsulated glucosesensitive polymer complex micelle (CM), which was selfassembled by block copolymer PEG-b-p (Asp-co AspPBA) and glycosaminoglyceride polymer $\mathrm{p}$ (Asp-coAspGA-co -AspNTA), forming a composite micelle with PEG shell and crosslinking core composed of PBA/glucose complex. The characteristics of the micelles are as 
follows: (1) the nitrogen tri acetic acid (NTA) group of glycopolymer based on glucosamine (GA)/n-aminotriacetic acid (NTA) binding to insulin specifically through chelating zinc ion, which greatly increases the insulin load; (2) the glucose partial pressure pulse release of insulin shows long-term stability under physiological conditions. Finally, in the type I diabetic mice model, the NTA-CM-INS group showed a long-term hypoglycemic effect, which was better than that of the PBS (PBS-INS) group and non-NTA-modified (CM-INS) group. This longterm effect could be attributed to the chelation of $\mathrm{Zn}$ by NTA-modified composite micelles, and the hypoglycemia caused by the sudden release of insulin can be avoided. Typically, this is an effective method to encapsulate insulin and control blood glucose in diabetic patients by releasing insulin on demand.

However, the polyamino acid-based insulin-glucose micelle core is biodegradable, which greatly reduces its stability and severely affects the protection of insulin. Therefore, most of the micelles cannot effectively protect insulin from the degradation of proteases in vivo, which greatly reduces the degradation of insulin in vivo, thus affecting its efficacy. Subsequently, Wu et al ${ }^{87}$ prepared glucose-loaded glucose-reactive composite micelles (CMs), a copolymer modified with PBA, PEG-b-P (Aspco-AspPBA), and glucosamine (GA)/n-aminotriacetic acid (NTA)-functionalized block copolymer PNIPAMb-P (Asp-co-AspGA-co-AspNTA), which is selfassembled because the CMs have a PEG/PNIPAM mixed shell. This assembly protects the plasma and enhances the blood-glucose-lowering activity in the body. CMs have PEG/PNIPAM-mixed shell and PBA/ GA complex crosslinked nucleus, which can be decomposed at high sugar concentration $(5 \mathrm{~g} / \mathrm{L})$ and stabilized at low concentration $(1 \mathrm{~g} / \mathrm{L})$. The NTA group of CMs significantly increases insulin loading by chelating zinc ions into insulin-specific binding. Importantly, the PNIPAM chain in the mixed shell collapses at $37^{\circ} \mathrm{C}$ and forms hydrophobic domains around the micellar nuclei, which can significantly protect the micellar nuclei and the encapsulated insulin from attack by external proteases, and in animal studies, NTA chelated CMs had the long-term hypoglycemic effect, which is superior to insulin-loaded single micelles without PNIPAM and PBS buffer ( $\mathrm{pH}$ 7.4) in type I diabetic mice. Therefore, such CMs are expected to become potential candidates for insulin release in the treatment of diabetes.

\section{Multifunctional-Combined PBA-Containing Biomaterials}

With the further development of the research, scientists found that the single glucose stimulation response system does not meet the needs of some fields in the complex human environment. In order to further improve the drug release performance, researchers have developed novel double stimulation and multi-stimulus-response polymer nanoparticles to respond to the combination of two or more signals. This multi-functional carrier can better adapt to the environment in human body and realize the intelligent release of drugs.

Cui et al ${ }^{88}$ used the RAFT method, based on the block copolymer poly ( $\mathrm{N}$-isopropyl acrylamide)-block poly (3-acrylamide-phenylboronic acid) (PNIPAM-b-PAPBA) $\mathrm{N}$-fluorescent coordination glucosamine poly(N-isopropyl acrylamide)/Eu(III)(GA-PNIPAM)/Eu(III) as raw materials, the structure and properties of the block copolymers were studied, and novel temperature-, $\mathrm{pH}$-, and glucosesensitive polymer nanoparticles were prepared by selfassembly. At the appropriate $\mathrm{pH}$ and temperature, the nanoparticles could be regulated by their collapse or glucose-induced swelling, with an average kinetic radius of about $80 \mathrm{~nm}$ and excellent fluorescence properties. The results of the MTT assay showed that when the concentration range of the nanocarrier was $0.1-1000 \mu \mathrm{g} / \mathrm{mL}$, there was no obvious cytotoxicity at any time of exposure.

Díez et al $^{89}$ designed an intelligent glucose-responsive nanosystem for insulin delivery based on the combination of ultrasound-driven nanomotors and enzyme-based sensing effector units. Insulin release was controlled by a $\mathrm{pH}-$ responsive PBA-glucose oxidase (GOx) supramolecular nanovalve coupled to the mesoporous silicon dioxide by a gold nanowire (AuNW) motor. The process of insulin release from the moving nanomachine is mainly glucosemediated and $\mathrm{pH}$-dependent. When the concentration of glucose in the environment increases, the induced protonation of the PBA group triggers the opening of the $\mathrm{pH}$ drive gate and the opening of the insulating nano valve (Figure 10). In addition to the $\mathrm{pH}$-dependent release, the enzymatically generated $\mathrm{H}_{2} \mathrm{O}_{2}$ may also participate in benzene-mediated cleavage of the $\mathrm{C}-\mathrm{B}$ bond of the boric acid part that promotes the overall release of insulin. Under the magnetic field, the effective motion of MS-Au nanomotor significantly accelerates the release of insulin. The combination of the nanoparticle carrier, the gated insulin nanoparticle container, and the 


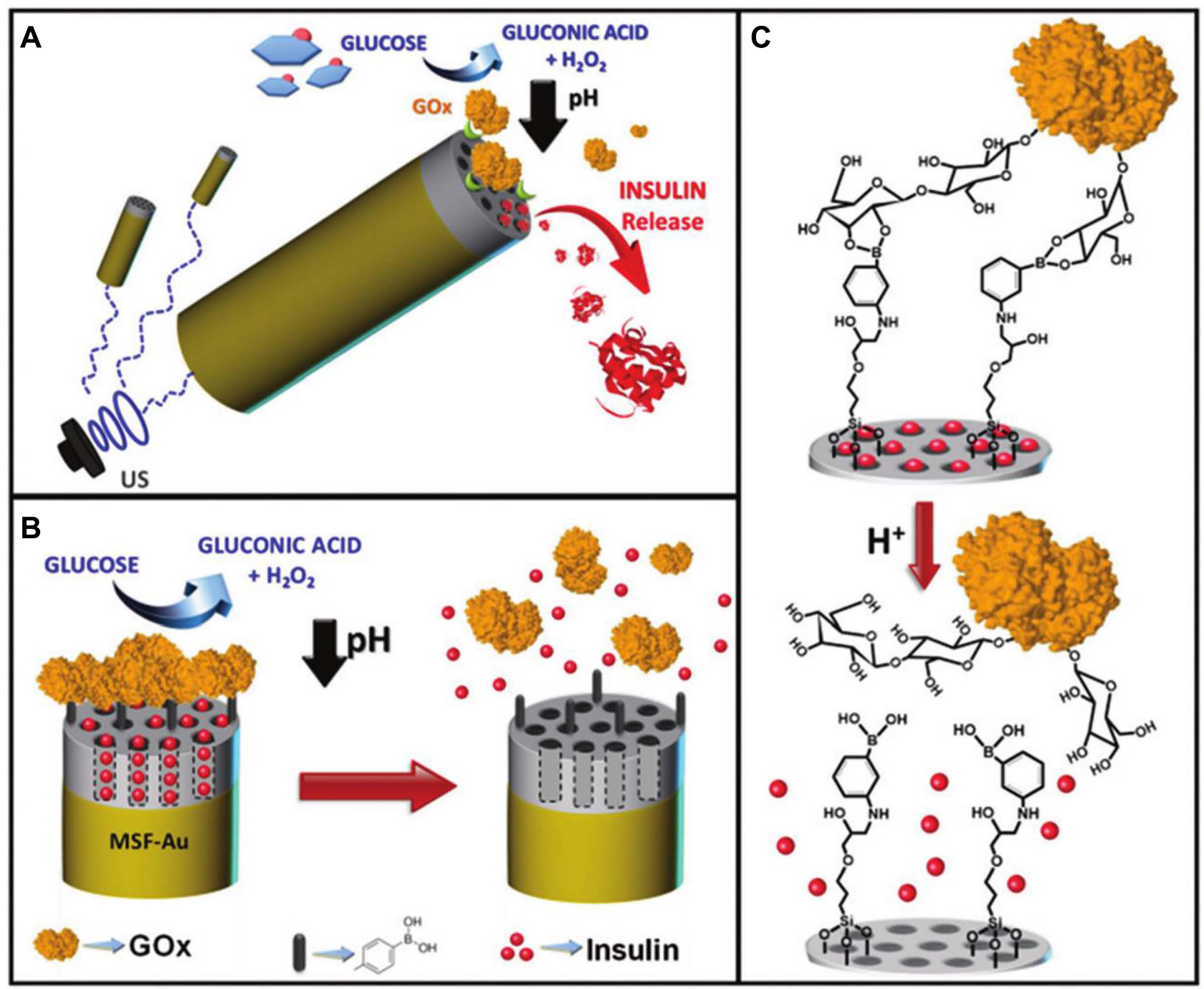

Figure 10 (A) Schematic illustration shows the pH-triggered insulin release nanomachine approach based on US-propelled mesoporous silica (MS)-Au nanomotors. (B) Glucose responsive gated insulin-containing nanocontainers. Steps involved in the insulin release mechanism: PBA-functionalized MS segment is capped with pH-sensitive nanovalves based on the GOx gating trigger molecule that leads to the autonomous insulin delivery in the presence of glucose. (C) Protonation of the PBA groups induces the opening of the $\mathrm{pH}$-driven gate and uncapping of the In-loaded nanovalves.

Notes: Reprinted from Díez P, Esteban-Fernández de Ávila B, Ramírez-Herrera DE et al Biomedical nano-motors: efficient glucose-mediated insulin release. Nanoscale. 2017;9(38): 14307-I43।I. Copyright (C) (2017), with permission from RSC Pub. ${ }^{89}$

movement of the glucose response nanoparticle valve is promising for improving the treatment of diabetes.

\section{Others}

Tong et $\mathrm{al}^{90}$ designed a dual-reaction insulin release device that combines glucose and $\mathrm{H}_{2} \mathrm{O}_{2}$-reactive polymer vesicles (PVs) and transdermal microneedles (MNs). PVs are selfassembled triblock copolymers composed of PEG, poly (phenylboronic acid) (PPBA, glucose-sensitive block), and polyphenylborate (PPBEM, $\mathrm{H}_{2} \mathrm{O}_{2}$-sensitive area). After loading insulin and glucose oxidase, the drugloaded PVs showed basal insulin release and promoted insulin release in the hyperglycemic state. The release rate of insulin responds rapidly to the increase in glucose and can be further promoted by added GOx, generating $\mathrm{H}_{2} \mathrm{O}_{2}$ at high sugar levels, further breaking the chemical connection of the PBEM group. This modification method provides long-term and glucose-mediated insulin activity, thereby reducing the number of administrations and improving the accuracy of insulin therapy for blood glucose control. Similarly, Liu et $\mathrm{al}^{91}$ fabricated glucose/ $\mathrm{H} 2 \mathrm{O} 2$ dual-response polymeric micelles for selfregulated insulin delivery. The polymeric micelles were self-assembled by poly(ethylene glycol)-block-poly (amino phenylboronic ester)(PEG-b-PAPBE), where the hydrophilic PEG offered the shell and the hydrophobic 
PAPBE endowed the polymeric micelles with the dualsensibility to glucose and $\mathrm{H} 2 \mathrm{O} 2$. Compared with free insulin or micelles carrying insulin alone, subcutaneous injection of insulin/GOx-loaded polymer micelles into diabetic mice has a better hypoglycemic effect in vivo. Further shows that this kind of polymeric micelle with glucose and $\mathrm{H}_{2} \mathrm{O}_{2}$ dual-responsiveness provides a promising approach for diabetes therapy.

Gold nanoclusters (GNCs), as a new type of gold nanomaterials, compared to traditional nano-carrier materials, have an ultra-small size $(2-3 \mathrm{~nm})$, good biocompatibility, easy surface modification, and high loading capacity of the biological effect of the dose. ${ }^{92,93}$ Zhang et $\mathrm{al}^{94}$ developed a GNC-based glucose-responsive insulin release system for blood glucose control in diabetic patients. GNCs are modified using 4-carboxyphenylboronic acid (PBA) or 4-carboxy-3-fluorophenylboronic acid (FPBA) molecules as response factors, and then, insulin is grafted onto the surface of GNCs to construct glucosesensitive insulin release systems GNC -PBA-Ins or GNCFPBA-Ins complex, respectively. These systems improve the release efficiency of insulin in response to blood sugar levels and regulate the blood sugar levels of type 1 diabetic mice within the normal blood sugar range for $48 \mathrm{~h}$, mimicking the function of the natural pancreas in the body.

\section{Future Perspective}

With the development of glucose-responsive insulin delivery systems, intelligent polymer delivery systems establish a link between therapeutic needs and drug delivery. The polymer delivery system based on PBA and its derivatives is a self-regulating drug-controlled release system because it can reduce the fluctuation of blood sugar and the number of insulin injections, improve patient compliance, and reduce the occurrence of complications; this renders that the system has a great prospect in the treatment and application of diabetes.

In this study, the research progress of the PBA-based glucose-sensitive drug delivery system is reviewed, the advantages and disadvantages of the combination of PBA and environmentally sensitive materials are analyzed. As deduced from our summary, although great progress has been made in the development of glucose-sensitive materials with different environmental responses based on PBA; however, there are still several issues: (1) the main problem is still how to maintain the activity of the glucose-responsive molecule under the physiological condition of the human body and realize the continuous glucose response behavior.
Several investigators have improved the glucose response ability of PBA based on glucose biomaterial under physiological conditions; however, the study of glucose-responsive drug delivery system with multienvironmental factors still needs to be strengthened; (2) the development of polymer materials with good biocompatibility, easy degradation, sensitivity reaction, and easy preparation is also the problem that the glucose-sensitive insulin-controlled release system needs to be resolved. The current biocompatibility testing for PBA and its derivatives is mostly cytotoxic, but the researchers should also look at the hemolytic properties of the material and its degradation in vivo. Also, the polymer with simple structure and easy preparation realizes the precise delivery of the drug easily when controlling the drug release. In terms of innovation: (1) Most of the current methods of administration are by injection. Can it be combined with other methods of administration to be less detrimental and more acceptable to the patient? This may further increase people's acceptance and compliance, such as subcutaneously, pulmonary, and orally. (2) Lowering the $\mathrm{pH}$ of PBA to be closer to the internal environment of the human body and making it sensitive to glucose is also the focus of further research. (3) In addition to the unstable blood sugar of diabetes, the long-term micro-inflammatory state of the patient is also the main reason for the progression of diabetes complications. In recent years, Chinese medicine monomers have been rewarded for improving the microinflammatory state of diabetic patients. Therefore, in addition to the combined use of PBA and blood-sugar-lowering drugs, can Chinese medicine monomers be combined with them to reduce blood sugar while improving diabetes patients' microinflammatory state?

It is speculated that the new self-regulated insulin controlled-release system based on phenylboronic acid will meet a series of conditions, such as good biocompatibility, easy degradation, and high glucose sensitivity under physiological conditions that will be beneficial to the majority of diabetic patients.

\section{Acknowledgment}

This investigation was supported by the grant from the Key Laboratory of Microcosmic Syndrome Differentiation, Education Department of Yunnan Project (20190720); Yunnan Province Applied Basic Research Project (2019FF002 (-055)).

\section{Disclosure}

The authors report no conflicts of interest in this work. 


\section{References}

1. Schmidt AM. Highlighting diabetes mellitus: the epidemic continues. Arterioscler Thromb Vasc Biol. 2018;38(1):e1-e8. doi:10.1161/ ATVBAHA.117.310221

2. Mantovani A, Scorletti E, Mosca A, et al. Complications, morbidity and mortality of nonalcoholic fatty liver disease. Metabolism. 2020;111S:154170. doi:10.1016/j.metabol.2020.154170

3. Buse JB, Wexler DJ, Tsapas A, et al. 2019 update to: management of hyperglycemia in type 2 diabetes, 2018. A consensus report by the American Diabetes Association (ADA) and the European Association for the Study of Diabetes (EASD). Diabetes Care. 2020;43 (2):487-493. doi:10.2337/dci19-0066

4. Karimi S, Jaafari A, Ghamari M, et al. A comparison of type ii diabetic patients with healthy people: coping strategies, hardiness, and occupational life quality. Int J High Risk Behav Addict. 2016;5 (1):e24169. doi:10.5812/ijhrba.24169

5. Li HP, He JL, Zhang MZ, et al. Glucose-sensitive polyphosphoester diblock copolymer for an insulin delivery system. ACS Biomater. 2020;6(3):1553-1564. doi:10.1021/acsb.iomaterials.9b01817

6. Wang Y, Fan Y, Zhang M, et al. Glycopolypeptide nanocarriers based on dynamic covalent bonds for glucose dual-responsiveness and self-regulated release of insulin in diabetic rats. Biomacromolecules. 2020;21(4):1507-1515. doi:10.1021/acs.biomac.0c00067

7. Chai Z, Dong H, Sun X, et al. Development of glucose oxidase-immobilized alginate nanoparticles for enhanced glucose-triggered insulin delivery in diabetic mice. Int $J$ Biol Macromol. 2020;159:640-647. doi:10.1016/j.ijbiomac.2020.05.097

8. Hashemi N, Valk T, Houlind K, et al. Insulin-based infusion system: preliminary study. J Diabetes Sci Technol. 2019;13(5):935-940. doi:10.1177/1932296818821349

9. Zhao L, Xiao C, Wang L, et al. Glucose-sensitive polymer nanoparticles for self- regulated drug delivery. Chem Commun (Camb). 2016;52(49):7633-7652. doi:10.1039/c6.cc02202b

10. JJ KIM, PARK K. Immobilization of ConcanavalinAto glucose-containing polymers. Macromol Symp. 2001;172(1):95-102. doi:10.1002/1521-3900(200107)172

11. Wu Q, Wang L, Yu H, et al. Organization of glucose-responsive systems and their properties. Chem Rev. 2011;111(12):7855-7875. doi: $10.1021 / \mathrm{cr} 200027 \mathrm{j}$

12. Ma R, Yang H, Li Z, et al. Phenylboronic acid-based complex micelles with enhanced glucose-responsiveness at physiological $\mathrm{pH}$ by complexation with glycopolymer. Biomacromolecules. 2012;13 (10):3409-3417. doi:10.1021/bm3012715

13. Wang QX, Wang HZ, Chen QB, et al. Glucose-Triggered Micellization of Poly(ethyleneglycol)-b-poly(N-isopropylacrylamideco-2-(acrylamido) henylboronic acid) Block Copolymer. ACS Appl Polym Mater. 2020;2(9):3966-3976. doi:10.1021/acsapm.0c00635

14. Zhong YH, Song B, He D, et al. Galactose-based polymer-containing phenylboronic acid as carriers for insulin delivery. Nanotechnology. 2020;31(39):395601. doi:10.1088/1361-6528/ab9e26

15. Guo H, Li H, Gao J, et al. Phenylboronic acid-based amphiphilic glycopolymeric nanocarriers for in vivo insulin delivery. Polym Chem. 2016;7(18):3189-3199. doi:10.1039/C6PY00131A

16. Elshaarani T, Yu H, Wang L, et al. Chitosan reinforced hydrogels with swelling-shrinking behaviors in response to glucose concentration. Int $J$ Biol Macromol. 2020;161:109-121. doi:10.1016/j. ijbiomac.2020.06.012

17. Mandal D, Mandal SK, Ghosh M, et al. Phenylboronic acid appended pyrene-based low-molecular-weight injectable hydrogel: glucose-stimulated insulin release. Chemistry. 2015;21 (34):12042-12052. doi:10.1002/chem. 201501170

18. Webber MJ, Anderson DG, Langer R. Engineering synthetically modified insulin for glucose-responsive diabetes therapy. Expert Rev Endocrinol Metab. 2015;10(5):483-489. doi:10.1586/ 17446651
19. Akira M, Syuhei I, Atsushi H, et al. Glucose-responsive polymer bearing a novel phenylborate derivative as a glucose-sensing moiety operating at physiologi -cal $\mathrm{pH}$ conditions. Biomacromolecules. 2003;4(5):1410-1416. doi:10.1021/bm0345413

20. Ma R, Shi L. Phenylboronic acid-based glucose-responsive polymeric nanoparticles: synthesis and applications in drug delivery. Polym Chem. 2014;5:1503-1518. doi:10.1039/C3PY01202F

21. Wang Y, Chai Z, Ma L, et al. Fabrication of boronic acid-functionalized nanoparticles via boronic acid-diol complexation for drug delivery. $R S C$ Adv. 2014;4(96):53877-53884. doi:10.1039/C4RA05034G

22. Matsumoto A, Ishii T, Nishida J, et al. A synthetic approach toward a self-regulated insulin delivery system. Angew Chem Int Ed Engl. 2012;51(9):2124-2128. doi:10.1002/anie.201106252

23. Watahiki R, Sato K, Suwa K, et al. Multilayer films composed of phenylboronic a cid-modified dendrimers sensitive to glucose under physiological conditions. J Mater Chem B. 2014;2(35):5809-5817. doi: $10.1039 / \mathrm{c} 4 \mathrm{tb} 00676 \mathrm{c}$

24. Li Q, Lü C, Liu Z. Preparation and characterization of fluorophenylboronic acid-functionalized monolithic columns for high affinity capture of cis-diol containing compounds. $J$ Chromatogr A. 2013;1305:123-130. doi:10.1016/j.chroma.2013.07.007

25. Wang B, Ma R, Liu G, et al. Glucose-responsive micelles from self-assembly of poly(ethylene glycol)-b-poly(acrylic acid-coacrylamidophenylboronic acid) and the controlled release of insulin. Langmuir. 2009;25(21):12522-12528. doi:10.1021/la901776a

26. Foss AC, Goto T, Morishita M, et al. Development of acrylic-based copolymers for oral insulin delivery. Eur $J$ Pharm Biopharm. 2004;57(2):163-169. doi:10.1016/S0939-6411(03).00145-0

27. Peng CL, Yang LY, Luo TY, et al. Development of $\mathrm{pH}$ sensitive-(diisopropylamino)ethyl methacrylate based nanoparticles for photodynamic therapy. Nanotechnology. 2010;21(15):155103. doi:10.1088/0957-4484/21/15/15.5103

28. Usman A, Zia KM, Zuber M, et al. Chitin and chitosan based polyurethanes: A review of recent advances and prospective biomedical applications. Int $J$ Biol Macromol. 2016;86:630-645. doi:10.1016/j.ijbiomac.2016.02.004

29. Dodane V, Vilivalam VD. Pharmaceutical applications of chitosan. Pharm SCI Technol To. 1998;1:246-253. doi:10.1016/S1461-5347(98)00059-5

30. Ma R, Wang B, Sun P, et al. B 3Q MAS NMR study on glucose-responsive micelles self-assembled from PEG-b-P(AA-co AAPBA). Chin J Chem. 2014;32:97-102. doi:10.1002/cjoc.20130.0920

31. Gaballa H, Theato P. Glucose-responsive polymeric micelles via boronic acid- diol complexation for insulin delivery at neutral pH. Biomacromolecules. 2019;20(2):871-881. doi:10.1021/acs. biomac. $8 \mathrm{~b} 01508$

32. Seno M, Yoshida K, Sato K, et al. $\mathrm{pH}$ - and sugar-sensitive multilayer films composed of acid (PBA)-modified poly(allylamine hydrochloride) (PBA-PAH) and poly(vinyl alcohol) (PVA): A significant effect of PBA content on the film stability. Mater Sci Eng C Mater Biol Appl. 2016;62:474-479. doi:10.1016/j.msec.2016.02.005

33. Xiaoqin Q, Wenping W, Wentao K, et al. Organic-inorganic hybrid hollow mesoporo -us organosilica nanoparticles for efficient ultrasound-based imaging and controlled drug release. J Nanomater. 2014. doi:10.1155/2014/972475

34. Li J, Qin X, Yang Z, et al. A novel mesoporous silica nanosphere matrix for the immobilization of proteins and their applications as electrochemical biosensor. Talanta. 2013;104:116-121. doi:10.1016/ j.talanta.2012.11.038

35. Tan L, Yang MY, Wu HX, et al. Glucose- and pH-responsive nanogated ensemble based on polymeric network capped mesoporous silica. ACS Appl Mater Interfaces. 2015;7(11):6310-6316. doi:10.1021/acsami.5b006.31

36. Wang B, Yoshida K, Sato K, et al. Phenylboronic acid-functionalized layer- by-layer assemblies for biomedical applications. Polymers (Basel). 2017;9(6):202. doi:10.3390/polym9060202 
37. De Geest BG, Jonas AM, Demeester J, De Smedt SC. Glucoseresponsive polyelectrolyte capsules. Langmuir. 2006;22 (11):5070-5074. doi:10.1021/la053368o

38. Sato K. Development of functional multilayer nanofilms and microcapsules based on layer-by-layer deposition techniques. Yakugaku Zasshi. 2015;135(9):1029-1035. doi:10.1248/yakushi.15-00182

39. Zhang SB, Chu LY, Xu D. Poly (N-isopropylacrylamide) -based comb-type grafted hydrogel with rapid response to blood glucose concentration change at physiological temperature. Polym Advan Technol. 2018;19(8):937. doi:10.1002/pat.1079

40. Qiu Y, Park K. Environment-sensitive hydrogels for drug delivery. Adv Drug Deliv Rev. 2001;53:321-339. doi:10.1016/s0169-409x(01)00203-4

41. Ron ES, Bromberg LE. Temperature-responsive gels and thermogelling polymer matrices for protein and peptide delivery. Adv Drug Deliv Rev. 1998;31(3):197-221. doi:10.1016/s0169-409x(97)00121-x

42. Wu JZ, Bremner DH, Li HY, et al. Synthesis and evaluation of temperature- and glucose-sensitive nanoparticles based on phenylboronic acid and N-vinylcaprolactam for insulin delivery. Mater Sci Eng C Mater Biol Appl. 2016;69:1026-1035. doi:10.1016/j. msec.2016.07.078

43. Li J, Yang L, Fan X, et al. Multi-responsive behaviors of copolymers bearing n-isopropylacrylamide with or without phenylboronic acid in aqueous solution. Polymers. 2018;10:3. doi:10.3390/polym10030293

44. Aslam K, Ahmed M, Javed A, et al. Semibath polymerization approach for one-pot synthesis of temperature- and glucose-responsive core-shell nanogel particles. $J$ Nanomater. 2018;5(18):1-9. doi:10.1155/2018/2180518

45. Yang $H$, Ma $R$, Yue $J$, et al. A facile strategy to fabricate glucose-responsive vesicles via a template of thermo-sensitive micelles. Polym Chem. 2015;6(20):3837-3846. doi:10.1039/C5PY00170F

46. Liu G, Ma R, Ren J, et al. A glucose-responsive complex polymeric micelle enabling repeated on-off release and insulin protection. Soft Matter. 2013;9(5):1636-1644. doi:10.1039/C2SM26690C

47. Wei Y, Zhang Z, Lu Y, Cheng Y. Advances of long-acting recombinant protein therapeutics. Chin J Biotechnol. 2018;34(3):360-368. doi: $10.13345 /$ j.cjb.17.0241

48. Davis CS, Fleming JW, Malinowski SS, et al. Ultra-long-acting insulins: A review of efficacy, safety, and implications for practice. J Am Assoc Nurse Pract. 2018;30(7):373-380. doi:10.1097/ JXX.0000000000000076

49. Gallegos Aragon K, Elmaoued AA, Pham NT, et al. Long-acting basal insulins: a review of the more recently approved agents. Cardiol Rev. 2019;27(5):260-266. doi:10.1097/CRD.0000000000000266

50. Priya James H, John R, Alex A, et al. Smart polymers for the controlled delivery of drugs - a concise overview. Acta Pharm Sin B. 2014;4(2):120-127. doi:10.1016/j.apsb.2014.02.005

51. Zhu LM, Wu JZ, Li HY, et al. Long-acting glucose-sensitive porous microspheres in layer-by-layer self-assembly and preparation method thereof. J Nanomater. 2016.

52. Abu Hashim II, Higashi T, Anno T, et al. Potential use of gamma-cyclodextrin polypseudorotaxane hydrogels as an injectable sustained release system for insulin. Int J Pharm. 2010;392(1-2):83-91. doi:10.1016/j.ijpharm.2010.03.026

53. Otsuka H, Uchimura E, Koshino H, et al. Anomalous binding profile of phenylboro -nic acid with $\mathrm{N}$-acetylneuraminic acid (Neu5Ac) in aqueous solution with varying pH. J Am Chem Soc. 2003;125 (12):3493-3502. doi:10.1021/ja021303r

54. Ivanov AE, Kumar A, Nilsang $S$, et al. Evaluation of boronate-containing polymer brushes and gels as substrates for carbohydrate-mediated adhesion and cultivation of animal cells. Colloids Surf B Biointerfaces. 2010;75(2):510-519. doi:10.1016/j. colsurf.b.2009.0.9.028

55. Liu H, Li Y, Sun K, et al. Dual-responsive surfaces modified with phenylboronic acid-containing polymer brush to reversibly capture and release cancer cells. J Am Chem Soc. 2013;135(20):7603-7609. doi:10.1021/ja401000m
56. Karimi F, Collins J, Heath DE, et al. Dynamic covalent hydrogels for triggered cell capture and release. Bioconjug Chem. 2017;28 (9):2235-2240.

57. Liu HW, Law WH, Lee LC, et al. Cyclometalated Iridium(III) bipyridine-phenylboronic acid complexes as bioimaging reagents and luminescent probes for sialic acids. Chem Asian J. 2017;12 (13):1545-1556. doi:10.1002/asia.201700359

58. Amaral AJ, Pasparakis G. Rapid formation of cell aggregates and spheroids induced by a "smart" boronic acid copolymer. ACS Appl Mater Interfaces. 2016;8(35):22930-22941. doi:10.1021/ acsami.6b07911

59. Ohno Y, Kawakami M, Seki T, et al. Cell adhesive character of phenylboronic acid-modified insulin and its potential as long-acting insulin. Pharmaceuticals (Basel). 2019;12(3):121. doi:10.1021/ acsami.6b07911

60. Han G, You CC, Kim BJ, et al. Light-regulated release of DNA and its delivery to nuclei by means of photolabile gold nanoparticles. Angew Chem Int Ed Engl. 2006;45(19):3165-3169. doi:10.1002/ anie. 200600214

61. Jiang G, Jiang T, Chen $\mathrm{H}$, et al. Preparation of multi-responsive micelles for controlled release of insulin. Colloid Polym Sci. 2015;293(1):209-215. doi:10.1007/s0039.6-014-3394-6

62. Salama A. Cellulose/calcium phosphate hybrids: new materials for biomedical and environmental applications. Int J Biol Macromol. 2019;127:606-617. doi:10.1016/j.ijbiomac.2.019.01.130

63. Peng H, Ning X, Wei G, et al. The preparations of novel cellulose/ phenylboronic acid composite intelligent bio-hydrogel and its glucose, pH-responsive behaviors. Carbohydr Polym. 2018;195:349-355. doi:10.1016/j.carbpol.2018.04.119

64. Linsley CS, Wu BM. Recent advances in light-responsive on-demand drug-delivery systems. Ther Deliv. 2017;8(2):89-107. doi:10.4155/ tde-2016-0060

65. Qiu M, Wang D, Liang W, et al. Novel concept of the smart NIR-light-controlled drug release of black phosphorus nanostructure for cancer therapy. Proc Natl Acad Sci U S A. 2018;115(3):501-506. doi:10.1073/pnas.1714421115

66. Yu W, Jiang G, Zhang Y, et al. Near-infrared light triggered and separable microneedles for transdermal delivery of metformin in diabetic rats. J Mater Chem B. 2017;5(48):9507-9513. doi:10.1039/ c7tb02236k

67. Zhang L, Li Y, Jin Z, et al. An NIR-triggered and thermally responsive drug delivery platform through DNA/copper sulfide gates. Nanoscale. 2015;7(29):1262-12614. doi:10.1039/ c5nr02767e

68. Liao G, Hu J, Chen Z, et al. Preparation, properties, and applications of graphene -based hydrogels. Front Chem. 2018;6:450. doi:10.3389/ fchem.2018.00450

69. Zhao W, Zhao Y, Wang Q, et al. Remote light-responsive nanocarriers for controlled drug delivery: advances and perspectives. Small. 2019;15(45):3190-3194. doi:10.1002/smll.201903060

70. Gooneh-Farahani S, Naimi-Jamal MR, Naghib SM. Stimuliresponsive graphene-incorporated multifunctional chitosan for drug delivery applications: a review. Expert Opin Drug Deliv. 2019;16 (1):79-99. doi:10.1080/17425247.2019.1556257

71. Jiang B, Qu Y, Zhang L, et al. 4-Mercaptophenylboronic acid functionalized graphene oxide composites: preparation, characterization and selective enrichment of glycopeptides. Anal Chim Acta. 2016;912:41-48. doi:10.1016/j.aca.2016.01.018

72. Basiruddin SK, Swain SK. Phenylboronic acid functionalized reduced graphene oxide based fluorescence nano sensor for glucose sensing. Mater Sci Eng C Mater Biol Appl. 2016;58:103-109. doi:10.1016/j.msec.2015.07.068

73. Teodorescu F, Oz Y, Quéniat G, et al. Photothermally triggered on-demand insulin release from reduced graphene oxide modified hydrogels. J Control Release. 2017;246:164-173. doi:10.1016/j. jconrel.2016.10.028 
74. Salvadó M, Reina JJ, Rojo J, et al. Topological defects in hyperbranched glycopolymers enhance binding to lectins. Chemistry. 2017;23(62):15790-15794. doi:10.1002/chem.201703432

75. Zheng C, Guo Q, Wu Z, et al. Amphiphilic glycopolymer nanoparticles as vehicles for nasal delivery of peptides and proteins. Eur J Pharm Sci. 2013;49(4):474-482. doi:10.1016/j.ejps.2013.04.027

76. Nagao M, Fujiwara Y, Matsubara T, et al. Design of glycopolymers carrying sialyl oligosaccharides for controlling the interaction with the influenza virus. Biomacromolecules. 2017;18(12):4385-4392. doi:10.1021/acs.biomac.7b01426

77. Guo Q, Zhang T, An J, et al. Block versus random amphiphilic glycopolymer nanoparticles as glucose-responsive vehicles. Biomacromolecules. 2015;16(10):3345-3356. doi:10.1021/acs. biomac. 5 b01020

78. Wu JZ, Bremner DH, Li HY, et al. Phenylboronic acid-diol crosslinked 6-O -vinylazeloyl-d-galactose nanocarriers for insulin delivery. Mater Sci Eng C Mater Biol Appl. 2017;76:845-855. doi:10.1016/j.msec.2017.03.139

79. Li D, Miao ZY, Bao C, et al. Assembly of silica nanoparticles based on stimuli-responsive covalent bonding between glycopolymers and poly(phenylboronic acid)s. Eur Polym J. 2020;135:109888. doi:10.1016/j.eurpolymj.2020.109888

80. Wei XS, Duan XZ, Ma Z, et al. Internalization mechanism of phenylboronic-acid-decorated nanoplatform for enhanced nasal insulin delivery. ACS Appl Bio Mater. 2020;3(4):2132-2139. doi:10.1021/acsabm.0c00002

81. Arokianathan JF, Ramya KA, Janeena A, et al. Non-proteinogenic amino acid based supramolecular hydrogel material for enhanced cell proliferation. Colloids Surf B Biointerfaces. 2020;185:110581. doi:10.1016/j.colsurfb.2019.110581

82. Ding J, Shi F, Xiao C, et al. One-step preparation of reductionresponsive poly(ethylene glycol)-poly (amino acid)s nanogels as efficient intracellular drug delivery platforms. Polym Chem. 2011;2857-2864.

83. Wang T, Yang X, Men J, et al. A near-infrared fluorescent probe based on boric acid hydrolysis for hydrogen peroxide detection and imaging in HeLa cells. Luminescence. 2020;35(2):208-214. doi:10.1002/bio. 3715

84. Zhao L, Ding J, Xiao C, et al. Glucose-sensitive polypeptide micelles for self-regulated insulin release at physiological $\mathrm{pH}$. J Mater Chem. 2012;22:12319-12328. doi:10.1039/C2JM31040F
85. Zhao L, Ding J, Xiao C, et al. Phenylboronic acid-functionalized polypeptide nanogel for glucose-responsive insulin release under physiological pH. J Control Release. 2015;213:e69. doi:10.1016/j. jconrel.2015.05.114

86. Li C, Huang F, Liu Y, et al. Nitrilotriacetic acid-functionalized glucose-responsive complex micelles for the efficient encapsulation and self-regulated release of insulin. Langmuir. 2018;34 (40):12116-12125. doi:10.1021/acs.la.ngmuir.8b0.2574

87. Wu G, Li C, Liu X, et al. Glucose-responsive complex micelles for self-regulated delivery of insulin with effective protection of insulin and enhanced hypoglycemic activity in vivo. Colloids Surf $B$ Biointerfaces. 2019;180:376-383. doi:10.1016/j.colsurfb. 2019.05.003

88. Cui G, Zhao K, You K, et al. Synthesis and characterization of phenylboronic acid-containing polymer for glucose-triggered drug delivery. Sci Technol Adv Mater. 2019;1(1):1-10. doi:10.1080/ 14686996.2019.1700394

89. Díez P, Esteban-fernández de Ávila B, Ramírez-Herrera DE, et al. Biomedicalnano-motors: efficient glucose-mediated insulin release. Nanoscale. 2017;9(38):14307-14311. doi:10.1039/c7nr05535h

90. Tong Z, Zhou J, Zhong J, et al. Glucose- and $\mathrm{h}_{2} \mathrm{O}_{2}$-responsive polymeric vesicles integrated with microneedle patches for glucose-sensitive transcutaneous delivery of insulin in diabetic rats. ACS App Mater Inter. 2018;10(23):20014-20024. doi:10.1021/ac. sami.8b04484

91. Liu XY, Li C, Lv J, et al. Glucose and h2o2 dual-responsive polymeric micelles for the self-regulated release of insulin. ACS Applied Bio Materials. 2020;3(3):1598-1606. doi:10.1021/acsabm.9b01185

92. He Z, Shu T, Su L, et al. Strategies of luminescent gold nanoclusters for chemo-/bio-sensing. Molecules. 2019;24(17):3045. doi:10.3390/ moleculees 24173045

93. Zheng Y, Lai L, Liu W, et al. Recent advances in biomedical applications of fluorescent gold nanoclusters. Adv Colloid Interface Sci. 2017;242:1-16. doi:10.1016/j.cis.2017.02.005

94. Zhang Y, Wu M, Dai W, et al. Gold nanoclusters for controlled insulin release and glucose regulation in diabetes. Nanoscale. 2019;11(13):6471-6479. doi:10.1039/c9n.r00668k
International Journal of Nanomedicine

\section{Publish your work in this journal}

The International Journal of Nanomedicine is an international, peerreviewed journal focusing on the application of nanotechnology in diagnostics, therapeutics, and drug delivery systems throughout the biomedical field. This journal is indexed on PubMed Central, MedLine, CAS, SciSearch ${ }^{\mathbb{}}$, Current Contents ${ }^{\mathbb{R}} /$ Clinical Medicine, $^{-}$
Journal Citation Reports/Science Edition, EMBase, Scopus and the Elsevier Bibliographic databases. The manuscript management system is completely online and includes a very quick and fair peer-review system, which is all easy to use. Visit http://www.dovepress.com/ testimonials.php to read real quotes from published authors. 